\title{
Electric Field Induced Biomimetic Transmembrane Electron Transport using Carbon Nanotube Porins as Bipolar Electrodes
}

\author{
Jacqueline M. Hicks ${ }^{1}$, Yun-Chiao Yao ${ }^{2}$, Sydney Barber ${ }^{3,4}$ Nigel Neate $^{5}$, Julie A. Watt ${ }^{1}$, Aleksandr Noy ${ }^{2,3}$, Frankie J. Rawson*1 \\ 1-Biodiscovery Institute, School of Pharmacy, Division of Regenerative Medicine and Cellular Therapies, University of \\ Nottingham, UK, NG7 2RD \\ 2- School of Natural Sciences, University of California Merced, USA, 95343 \\ Yun-Chiao Yao 3- Materials Science Division, Lawrence Livermore National Laboratory, USA, 94550 \\ 4-United States Naval Academy, USA, 21402 \\ 5-Nanoscale and Microscale Research Centre, University of Nottingham, UK, NG7 2RD
}

*Email: frankie.rawson@nottingham.ac.uk

\begin{abstract}
Cells modulate their homeostasis through the control of redox reactions via transmembrane electron transport systems. These are largely mediated via oxidoreductase enzymes. Their use in biology has been linked to a host of systems including reprogramming for energy requirements in cancer. Consequently, our ability to modulate membrane redox systems may give rise to opportunities to modulate underlying biology. The current work aimed to develop a wireless bipolar electrochemical approach to form on-demand electron transfer across biological membranes. To achieve this goal, we show that using membrane inserted carbon nanotube porins that can act as bipolar nanoelectrodes, we could control electron flow with externally applied electric fields across membranes. Before this work, bipolar electrochemistry has been thought to require high applied voltages not compatible with biological systems. We show that bipolar electrochemical reaction via gold reduction at the nanotubes could be modulated at low cell-friendly voltages, providing an opportunity to use bipolar electrodes to control electron flux across membranes. Our observations present a new opportunity to use bipolar electrodes to alter cell behavior via wireless control of membrane electron transfer.
\end{abstract}

\section{Keywords}

Carbon nanotube porins, trans plasma membrane electron transport systems, voltage-dependent anionselective channel bipolar electrochemistry, cell electrochemistry, membrane potential

\section{Introduction}

Trans-plasma membrane electron transport systems (TPMETs) are a ubiquitous family of proteins that facilitate the transport of electrons across membranes through redox cascades ${ }^{1}$. TPMETs consist of enzymes alone or in combination with naturally occurring electrochemical mediators which facilitate electron transfer ${ }^{2,3}$ and are present in all forms of life ${ }^{4}$. They are vitally important in cellular defence ${ }^{4,5}$, redox homeostasis, 
maintaining cellular energy levels and autophagy ${ }^{6}$. A ubiquitous example of an TPMET and one of the most well-studied is the NOX reductase family. Present in almost all cell types, NOX is involved in signaling and DNA regulation and is vital for maintaining the redox state of the cell. Unsurprisingly, malfunctioning NOX has been linked to numerous diseases and pathologies, 8 including neurodegenerative diseases and dementia'. Therefore, if we can devise techniques to artificially transport free electrons across biological membranes, it would open up new opportunities to modulate cell biology in the future. For example, if we can alter membrane electron transfer we have the opportunity to reprogramme cellular metabolism to restore cells' electrical communication to a normal state which, may be useful in developing new bioelectronic medicines. ${ }^{10}$, 11

Voltage-dependent anion-selective channels (VDAC's) ${ }^{6}$ are a subset of the TPMET family that act as switchable porins within the membrane for the transport of anions and have been shown to transfer electrons via redox, activated by a change in potential. VDACs are present in the plasma membrane in numerous cell types ${ }^{12}$ including neuron $\mathrm{s}^{13}$, remaining inactive until a change in the membrane potential is detected ${ }^{14,15}$. The need for VDACs to be 'activated' and have their pores opened explains how they are non-lethal, although the VDACS are thought to be involved in both ATP release and volume control ${ }^{16}$ of the cytosol playing a regulatory role in apoptosis. VDACS and TPMETs are up-regulated in various cancers $;{ }^{17}$ and are involved in cancer development and progression ${ }^{18,19}$. VDACs have also been shown to play a role in apoptosis within differentiated neurons ${ }^{20}$ and to be involved in amyloid beta toxicity in certain disease states including Alzheimers ${ }^{13}$. Designing biomimetic VDACs whose function can be controlled by external stimulus would be a key step in the treatment of such diseases.

The design and use of biomimetic membrane proteins (BMPs), including artificial TPMETs and VDACs, has drawn a lot of attention over the last decade ${ }^{21}$. The attraction of BMPs is twofold: first, they can be used for individual cellular measurements such as membrane potential and have proven to be extremely useful in optogenetics ${ }^{22,23}$ where light is used to manipulate ion channels for precise activation or inhibition of neuronal signals. Second, BMPs may be used in electroceutical therapies and not only for the detection of aberrant cellular behavior but also in its correction ${ }^{24-26}$. Biomimetic membrane proteins were recently shown by Ma et al. to be capable of correcting malfunctioning cells by injecting nanoparticles into mice, where those nanoparticles were able to bind to retinol photoreceptors and convert near IR light into the visible spectrum, restoring sight ${ }^{27}$. Nanoparticles and nanotubes are common building block BMPs ${ }^{28-30}$. However, for them to be useful they need to be functionalized to be water-soluble, active within biological media ${ }^{31,32}$ and able to be amphiphilic so they can insert and remain within the plasma membrane. These goals have been achieved with several approaches including protein cage $\mathrm{e}^{33}$, DNA origami ${ }^{34}$ and the attachment of ligands ${ }^{35}$.

An emerging BMP type-carbon nanotube porins (CNTPs) are artificial porins based on lipid-coated ultra-short carbon nanotubes that can self-insert into membranes ${ }^{35}$. They are similar in size to VDACs ${ }^{36}$ with diameters ranging from $0.7 \mathrm{~nm}-1.5 \mathrm{~nm}$ and with a concentration of charged groups at either terminus making the narrow $0.7 \mathrm{~nm}$ CNTPs appear 'closed', like VDACs, under normal biological conditions. Besides their ability to act as a channel for charged particles or water transport ${ }^{37,38}$, CNTPs are conductive ${ }^{39}$ and, potentially could act 
as wireless bipolar electrodes (BPEs). BPEs, placed within an electric field are polarised allowing for the reduction or oxidation of molecules at either terminus ${ }^{40,41}$; similar to how TPMETs/VDACs reduce oxidants extracellularly while oxidising reductants intracellularly. Typically, the potential experienced at the BPE will be a fraction of the potential applied across the two driving electrodes, dependent on the ratio between the length of the BPE and the distance between the driving electrodes ${ }^{42}$. It has been shown that for CNT BPES millimeters in length require driving potentials in the $\mathrm{kV}$ range are required for the potential felt at the CNT BPE terminus to be in the $1-10 \mathrm{~V}$ range ${ }^{41}$. Such a drastic potential drop would not be useable in biological systems. However, recently it has been shown that nano-BPEs that do not obey these rules can function with much lower driving potentials can be used ${ }^{43-45}$. This is because earlier research has failed to take account of capacitance increase at structures below $50 \mathrm{~nm}^{46}$ and the enhanced electric field around the nanoscale conductive objects

Herein we describe a nanotechnology-based route for controlling membrane electron transport. To investigate the use of CNTPs as BPEs and as artificial VDACs within biological systems (Figure 1), CNTPs were self-inserted into giant unilamellar vesicles (GUVs) which act as a simplified cellular model ${ }^{47}$ and can be studied using normal cell biology techniques ${ }^{48}$. GUVs were essential in the study of the function of CNTPs as nano-BPEs without the presence of native VDACs or other membrane proteins that may obscure results. After the initial studies in GUVs to ascertain the key parameters it was possible to investigate the CNTPs within a complex cellular system. Studies with a neuronal cell line (NG108-15) showed that CNTPs embedded within biological membranes can act as bipolar wireless electrodes and control localized redox events at cell-friendly voltages.

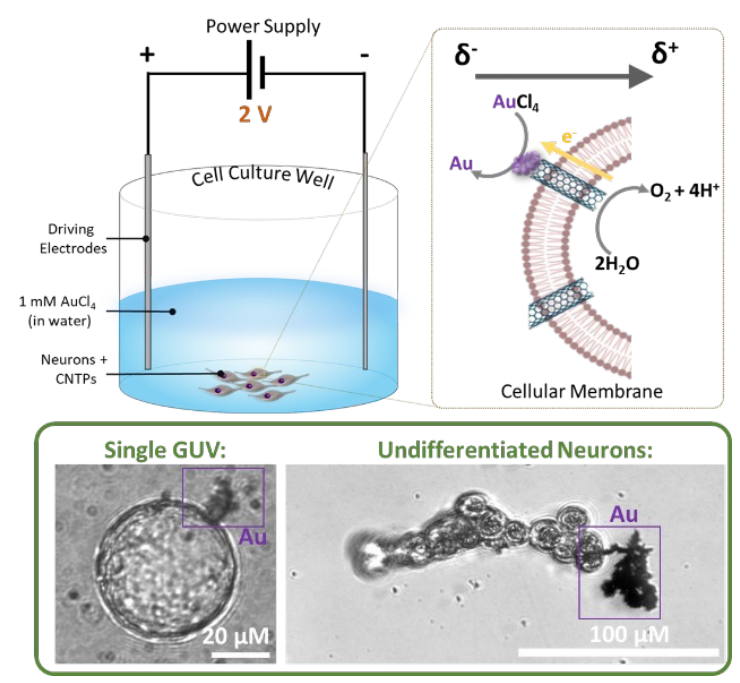

Figure 1 Summary schematic showing the experimental setup within a cell culture well of the driving electrodes and their placement on either side of the growth of NG108-15 undifferentiated neurons. CNTPS within neuronal membranes were polarised triggering the reduction of gold chloride to solid gold deposits on the CNTP terminus. Brightfield images below show this deposition on both a giant unilamellar vesicle (GUV) and the neurons.

\section{Results and Discussion}

We have inserted CNTPs into GUVs by adding lipid-coated CNTPs to the lipid mixture during the GUV preparation step. Before the incorporation of the CNTPs into GUVs, they were modified with the FITC based fluorophore, 6-aminofluorescein (6AF), via EDC/NHS carbodiimide coupling chemistry, to enable us to track the 
location of the CNTPs (see Figure 2). To separate modified CNTs the solution was passed through a sepharose gel column (CL-6B) to separate particles by size with fractions components identified by their absorption profiles (see Figure 2D). The covalent binding of the CNTPs to the 6AF fluorophore was then confirmed by FTIR and RAMAN spectroscopy (see Supporting Information).

GUVs containing both the 18:1 liss rhodamine PE and the CNTP-6AF were imaged with fluorescence microscopy (Error: Reference source not found 2.) These images show that the CNTPs are fully integrated within the GUV membrane with green fluorescence from the 6AF visible throughout the GUV population.
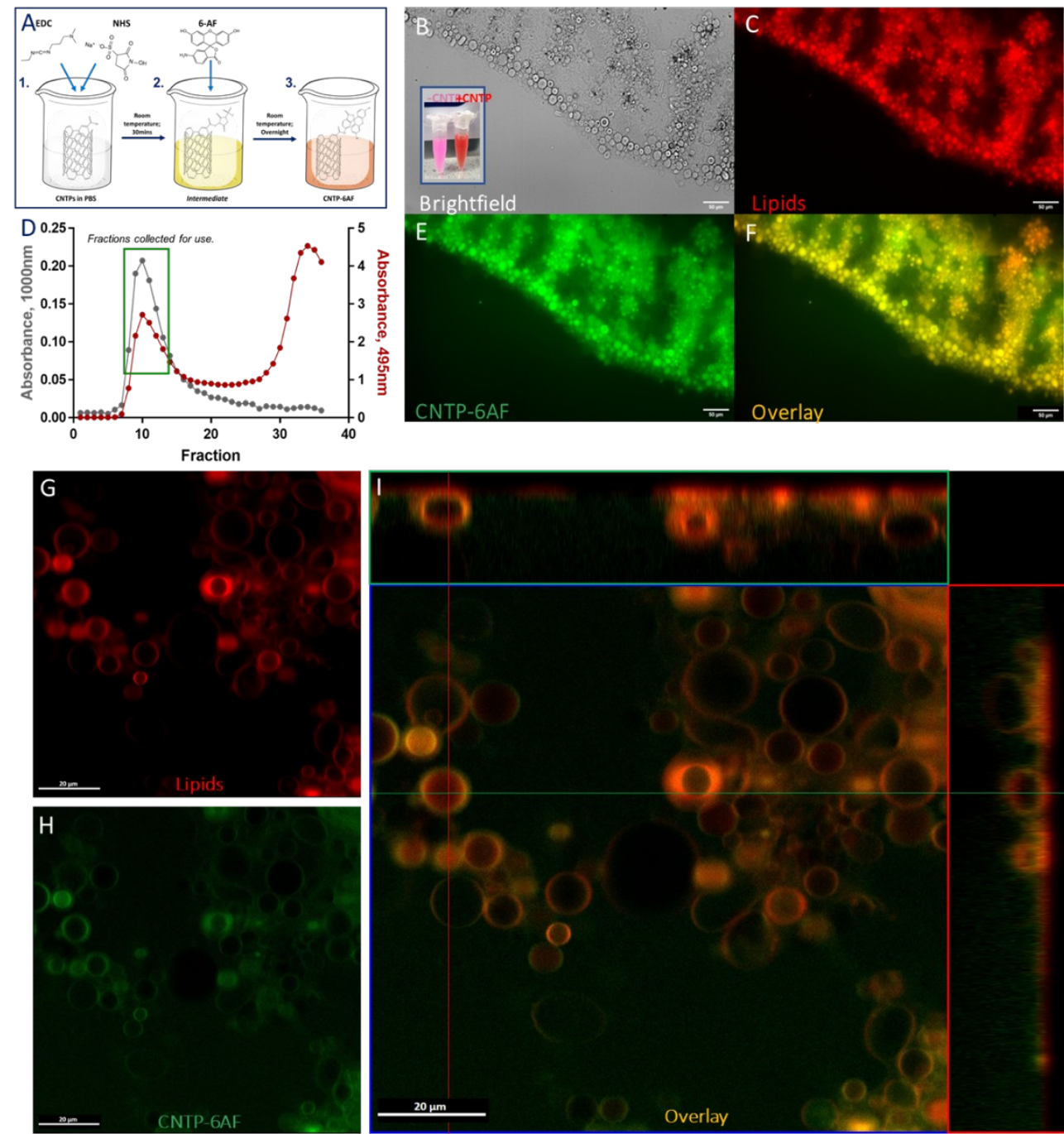

Figure 2: A\&D Modification of CNTPs with 6-aminofluorescein (6AF). A- Schematic of the reaction of CNTPs with 6AF via EDC/NHS chemistry. Step 1: solubilization of CNTPS with EDC (80mM) and NHS (20mM) which is left to react at room temperature for 30 minutes. Step 2: Addition of 6AF (final concentration $2.5 \mathrm{mM}$ ) which forms a yellow solution and is left overnight to react in the dark at room temperature. Step 3: Final product forms an orange solution. D- After separation of the product by size, gel-chromatography fractions absorbance is measured at 1000nm (left axis, CNTPs) and 495nm (right axis, 6AF). B-Brightfield microscopy of GUVS formed from rehydration. Inset Image- Shows difference in solution colors of the 18:1 Liss Rhod PE GUVs with and without the CNTP-6AF. C- Fluorescence microscopy of GUVS made with 18:1 Liss Rhod

PE and CNTP-6AF. Excitation/emission: 560/583nm, shows the 18:1 Liss Rhod PE lipid content of the GUVS. E-

Excitation/emission: 490/520nm, from the CNTP-6AF fluorophore. F- Overlay of the red and green channels. Scale bars are $50 \mu \mathrm{m}$. G-I Confocal microscopy of GUVS made with 18:1 Liss Rhod PE and CNTP-6AF. G- Excitation/emission: 561/624nm, shows the 18:1 Liss Rhod PE lipid content of the GUVS. H- Excitation/emission: 488/525nm, from the CNTP-6AF fluorophore.

I- Ortholog z-stack overlay of the red and green channels with cross-sectional panels. Scale bars are $20 \mu \mathrm{m}$. 
We used confocal microscopy imaging to investigate whether the CNTPs were integrated within the GUV outer membranes or whether they had been collected and pooled within the GUVs. A sample of GUVs imaged by confocal microscopy can be seen in Figure 2G-I. We observed halo type rings which define the GUV in the microscopy images (Figure 2G-I). These images show that CNTP-6AF molecules are localized to the outer membrane of the GUVs. This observation is also supported by the TEM images in the supporting information (Figure SI3).

To establish if we could use the CNTs as bipolar electrodes to facilitate membrane electron transfer we exposed the CNTPs modified GUVs to an electric field, causing the CNTs to polarize. This provides the thermodynamic driving force to facilitate redox reactions at the CNTPs without direct-wired contact to the feeder electrodes. In this experiment GUVs were submerged in a solution of $1 \mathrm{mM}$ gold chloride between two driving electrodes and electrochemical reduction of gold (eq 2) was used as a tool to visualize the nano BPEs activity. Figure 3 shows brightfield images of GUVs (with and without CNTP incorporation) after the application of a $\mathrm{DC}$ voltage for one hour in the presence and absence of $\mathrm{AuC}_{2}$.

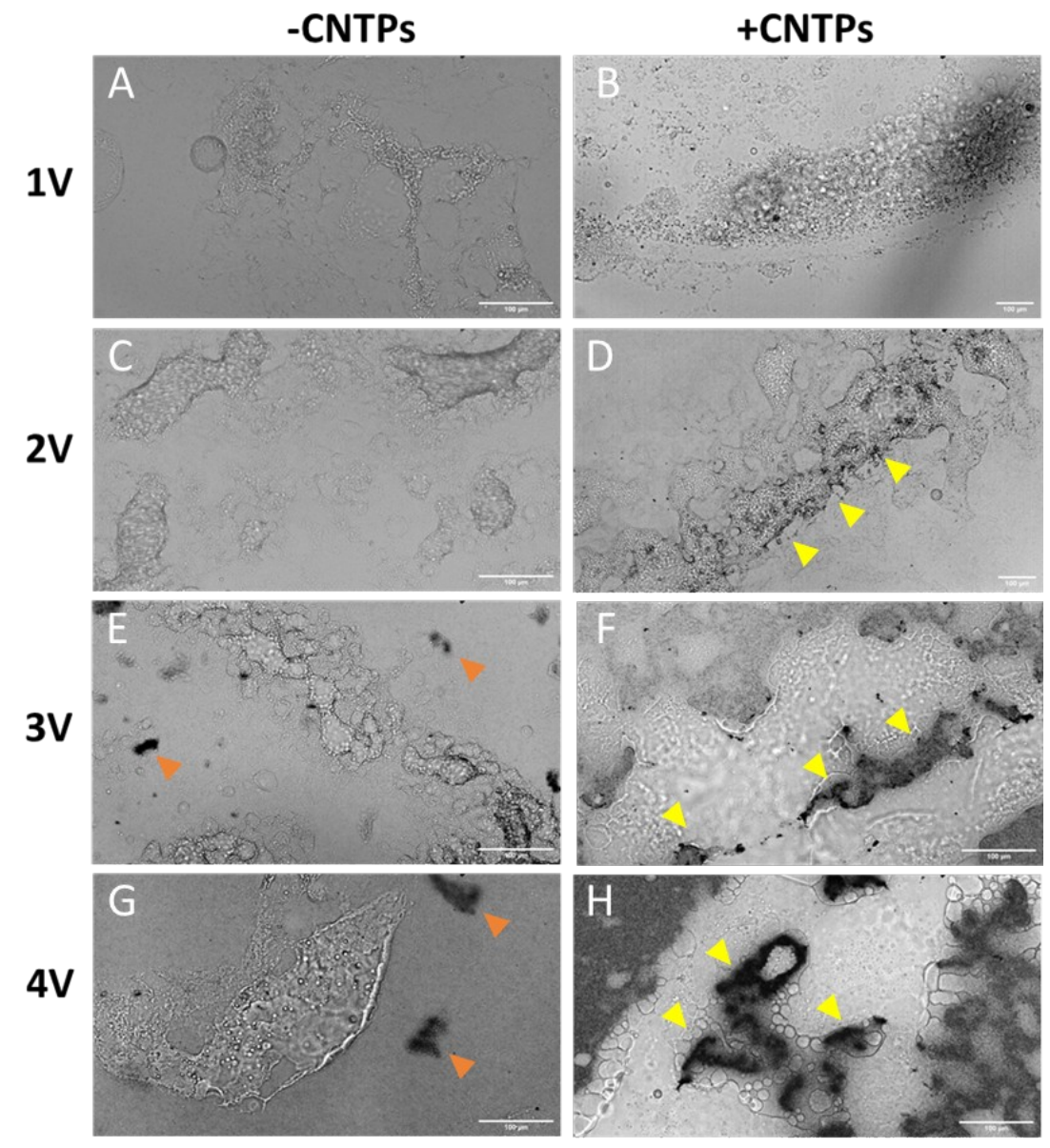

Figure 3: Brightfield images of GUVs with and without CNTPs incorporated and with varying DC voltages applied for 1 hour. GUVs were rehydrated with $20 \mu \mathrm{L}$ water for 15 minutes before being immersed in $1 \mathrm{ml} \mathrm{AuCl}{ }_{4}(1 \mathrm{mM})$. Panels $A, C, E$ and $G$ are without CNTPs. Panels B, D, F and H are with CNTPs. A \& B: 1 V, C \& D: 2 V, E \& F: 3 V, G \& H: 4 V. Scale bars show 100 $\mu \mathrm{m}$. Yellow arrows indicate the supposed reduced gold aggregates formed after application of voltage and attached to the lipid surface. Orange arrows indicate large, reduced gold aggregates formed at the driving electrodes that have broken off and are floating in solution while yellow arrows indicate gold reduced at the lipid surface. 
Figure 3 it can be seen that gold deposits become visible at GUV's modified with CNTPs after the application of $2 \mathrm{~V}$ for one hour and become larger when increased voltage is applied. On application of $4 \mathrm{~V}$ for one hour there is very little space left as it is occupied by the gold deposits. We then followed a time course of this process to further highlight the dynamic nature of the Au deposition process. Importantly, this only occurs on the application of the voltage and in the presence of CNTPs. Figure 4A shows how the gold deposits were distributed across the whole GUV spot after $4 \mathrm{~V}$ had been. The gold deposits are opaque so while imaging with an inverted light microscope they appear black. We subsequently plotted the image intensity values across the GUV spot (Figure 4D) that shows that the gold is preferentially reduced at one end of the GUV spot with a concentration gradient formed across the sample. Figure 4 shows snapshots of the lipids containing CNTPs at 30 minutes (Figure 4B-C) stimulation and 45 minutes stimulation (Figure $4 \mathrm{E}-\mathrm{F}$ ). The regions outlined highlight a specific region where the gold deposition growth can be seen. The CNTPs within the GUVs act as nucleation points for the electrochemically induced reduction and deposition. As time progresses with the application of a potential these deposits grow, as described in the literature ${ }^{41}$, and eventually break off allowing more gold reduction and deposits to grow. The gold deposition is evident at time points throughout the experiment (Figure 4) and is also seen in the time-lapse data of the gold aggregation (see supporting information (Figure SI 7)).

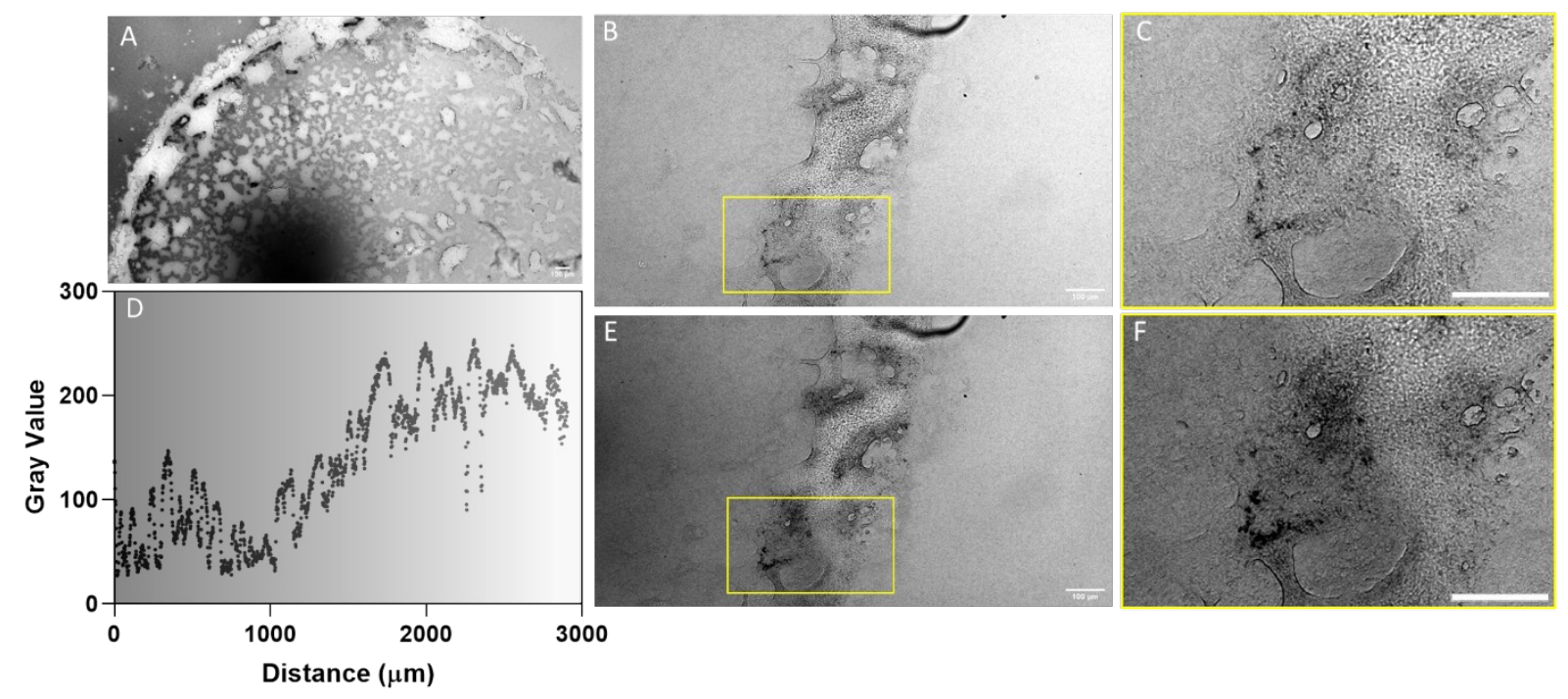

Figure 4: Brightfield images of GUVs with CNTPs incorporated. GUVs were rehydrated with $20 \mu$ l water for 15 minutes before submission in $1 \mathrm{ml} \mathrm{AuCl}_{4}(1 \mathrm{mM})$. A DC of 4 volts was applied. A- Lower magnification image showing the distribution of gold deposits across the GUV spot. B- Measured grayscale values across A. Panels B \& C are images taken 30 minutes after the beginning of stimulation. $E \& F$ of the same sample area imaged 45 minutes after the beginning of stimulation.

Right-hand panels are enlarged regions of the areas indicated in the left-hand panels. All scale bars are $100 \mu \mathrm{m}$.

We used energy dispersive $\mathrm{x}$-ray analysis (EDX) coupled with scanning electron microscopy (SEM) to further confirm gold deposition. Several different applied voltages were tested in samples made with and without CNTP incorporation as well as the original gold chloride solution. Multiple regions were randomly selected for imaging. To ensure the images were representative multiple regions were analyzed for each sample. Elemental reconstructions of carbon, gold and chlorine for the original gold chloride solution and a GUV sample exposed to voltage are shown in Figure 5. 

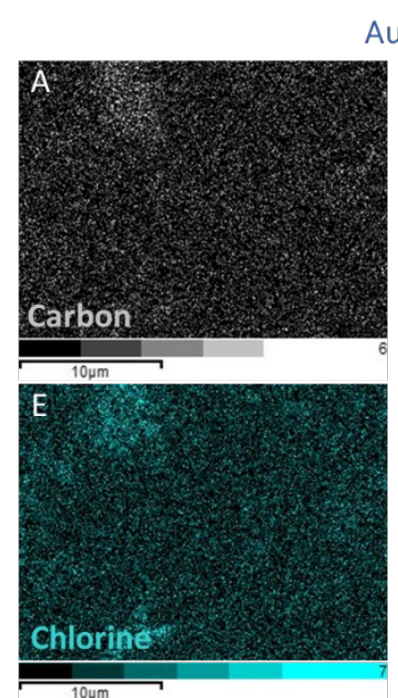

Gold

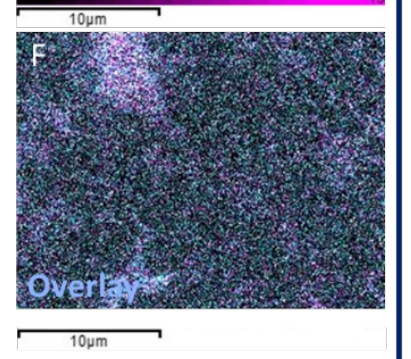

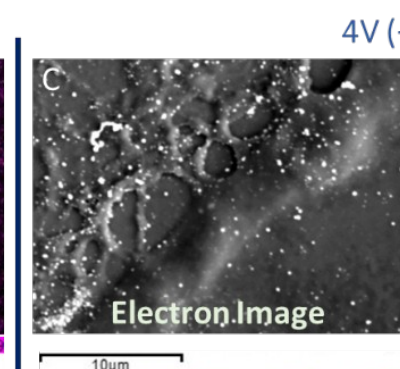

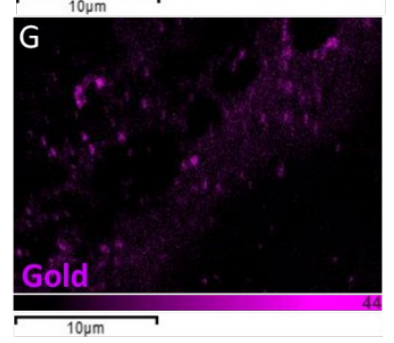

$4 \mathrm{~V}(+\mathrm{CNTP})$
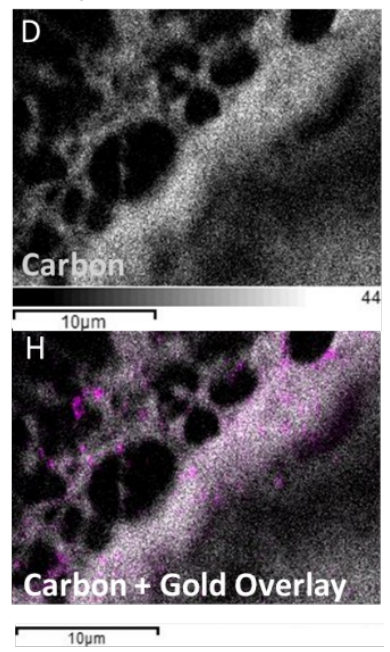

Figure 5: EDX analysis images. Panels $A, B, E \& F$ are from a sample of $A_{u C l}$ solution (1mM) dried under vacuum on a glass coverslip. Panels C, D, G\& H are from a sample of GUVs containing CNTPs on a glass coverslip, 3V (DC) applied for 1 hour in

a solution of $1 \mathrm{mM} \mathrm{AuCl}_{4}$ then dried under vacuum. Carbon ( $A \& C$ ), gold (magenta colored, $B \& D$ ) and chlorine (cyan colored, $E \& G$ ) elements were mapped and overlays plotted ( $F \& H)$. Intensity scale bars for each element are shown under each panel. Scale bars are $10 \mu \mathrm{M}$.

The original solution of gold chloride shows that the gold and chlorine co-localize as expected in the absence of an applied voltage. After, after application of a potential to the sample with GUVs containing CNTPs, concentrated deposits of gold appear with no corresponding chlorine detected. This observation supports the data from Figure 3 and Figure 4 and that the deposits visualized after application of voltage are indeed reduced gold.

Macro and micro-sized BPEs obey the rule that only a fraction of the applied potential is experienced at the BPE terminus. This behavior is defined by equation $1^{49-51}$ which explains the relationship between the potential at the BPE $\left(\Delta \mathrm{E}_{\text {elec }}\right)$, the length of the BPE $\left(\mathrm{I}_{\text {elec }}\right)$, the distance between the driving electrode $\left(\mathrm{I}_{\text {channel }}\right)$ and the driving potential $\left(E_{\text {tot }}\right)$. If nano-BPEs obeyed the same rules, to reach a potential of at least $1 \mathrm{~V}$ (to (required to reduce the gold, see equation 2) we would need to be applying at least $3 \mathrm{~V}$ if we considered the entire GUV spot as a BPE, or $1.2 \mathrm{kV}$ if we consider the CNTPs as individual BPES. As clearly neither of these is the case, it is important to better understand the nature of nano-BPEs. Finding the threshold potential for the gold reduction at the CNTPs was, therefore, an important first step in understanding how they work.

$$
\begin{aligned}
& \Delta E_{\text {elec }}=E_{\text {tot }}\left(\frac{l_{\text {elec }}}{l_{\text {channel }}}\right) \\
& \mathrm{E}^{\circ}=0.99 \mathrm{~V} \quad \mathrm{Au}(\mathrm{III}) \mathrm{Cl}_{4}^{-}+3 \mathrm{e}^{-} \text {à } \mathrm{Au}+4 \mathrm{Cl}^{-}
\end{aligned}
$$

By applying voltages for 1 hour at $0.1 \mathrm{~V}$ increments we observe it was discovered that gold was reduced at applied voltages as low as $1.5 \mathrm{~V}$. Moreover, with this low voltage, the GUVs did not completely fuse as seen in Figure 3, and growths of gold deposits could be seen clearly at individual GUVs (see Figure 6C) with Images showing the gold forming directly at the membrane of the GUV containing CNTPs. We wanted further evidence that wireless electrochemical deposition was occurring. Therefore, we monitored the signal from the 6-AF 
fluorophore attached to the CNTPs and observed changes in fluorescence by using optical microscopy on the application of a voltage. We observed that GUVs were polarised resulting in gold deposits at one end only that also quenched the CNTP fluorescence (see Figure 6 D-F). This observation further supports that the gold was being electrochemically deposited at the CNTP tip.

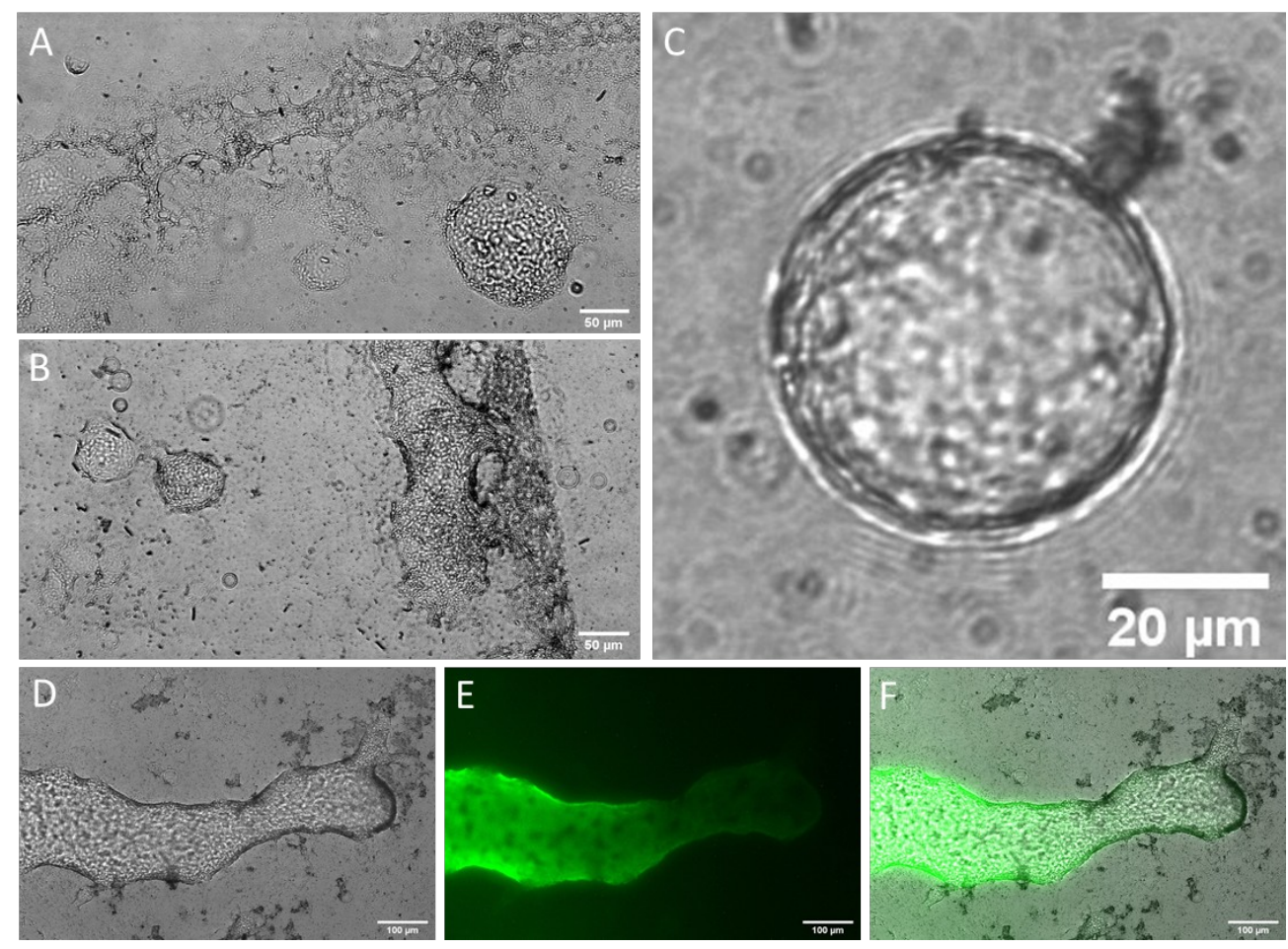

Figure 6 GUVs were stimulated with 1.5V DC for $1 \mathrm{hr}$ while submerged in $1 \mathrm{mM} \mathrm{AuCl} 4$ solution. (A) GUVs without CNTPs. (B) With CNTPs and (C) a zoomed image of a single GUV containing CNTPs after 1.5V application and visible gold deposition on the membrane. A\& B scale bars are $50 \mu \mathrm{m}$ and $C$ scale bar is $20 \mu \mathrm{m}$ in length.

We wanted to provide further mechanistic evidence to establish that direct bipolar electrochemical induced deposition of gold was occurring. To confirm that this deposition was not arising due to a mediated electrochemical process that was occurring due to electrochemically induced water splitting and radical formation that subsequently reduced the gold, we replaced the gold chloride solution with an equivalent solution of zinc chloride. This would establish if there was a potential relationship that would be indicative of the different standard potential of $\mathrm{Zn}\left(-0.76 \mathrm{~V}^{52}\right)$ versus $\mathrm{Au}\left(+0.99 \mathrm{~V}^{41}\right)$ (Fig SI6) while also confirming that low, cell-friendly voltages can be used with nano-BPEs for multiple, different redox reactions. If metal deposition was driven by radical formation during water splitting, then there would be no voltage difference observed with the applied feeder electrode potential. On the contrary, we observed that a significantly higher applied potential of $7 \mathrm{~V}$ at the feeder electrodes was required to observe reduced Zn (Fig SI6) versus the 1.5V that produced gold deposits. This result confirmed that the electrochemical mechanism is via direct bipolar electrochemical induced deposition and not an indirect electrochemical method.

We then wanted to show that CNTPs could act as BPEs in the cell membrane of NG108 cells and that electron transfer across the membrane via CNTPs could be modulated on-demand using wireless electrochemistry. We first provide evidence that CNTPs could be inserted into the membrane. We found that incubation of the 
CNTPs with the cells for four hours was sufficient to allow CNTPs to self-insert into the cellular membrane (Figure 7F). This behaviour is consistent with previous work that have shown lipid coating the CNTPS allows them to self-insert within the lipid membrane ${ }^{53}$. A control set of cells just incubated with PBS (see Figure 7B) showed similar changes in cellular morphology which we attribute to the CNTP insertion. On the addition of CNTPs to the cell, we wished to establish that the CNTPs did not affect cell viability. To assess viability after incubation with CNTPs in PBS buffer, cells were washed and the live cell stain NucBlue was added. Images of the cells after the CNTP addition show a substantial population of healthy cells (as indicated by the NucBlue fluorescent signal) left on the cell culture plate, with the viable cell percentage comparable to the PBS control. After incubation with either the PBS or CNTPs, cells were submerged (as before with the GUVs) in $1 \mathrm{mM} \mathrm{AuCl}{ }_{4}$ (in MilliQ water) and were stimulated for 1 hour at voltages between 1 and $4 \mathrm{~V}$. Due to the presence of proteins such as native VDACs within the cell membrane and their ability to reduce molecules once activated by voltage, there was a small amount of reduced gold at the membrane surface in all control samples as well (Figure 7G). This made it difficult to tell at the lowest voltage of $1.5 \mathrm{~V}$ if there were significantly more deposits on cells containing CNTPs which was occurring via bipolar electrochemistry. However, by $2 \mathrm{~V}$ there is a clear difference between the CNTP samples and the controls with deposits associated with the cell plasma membranes containing CNTPs (see Figure 7H).
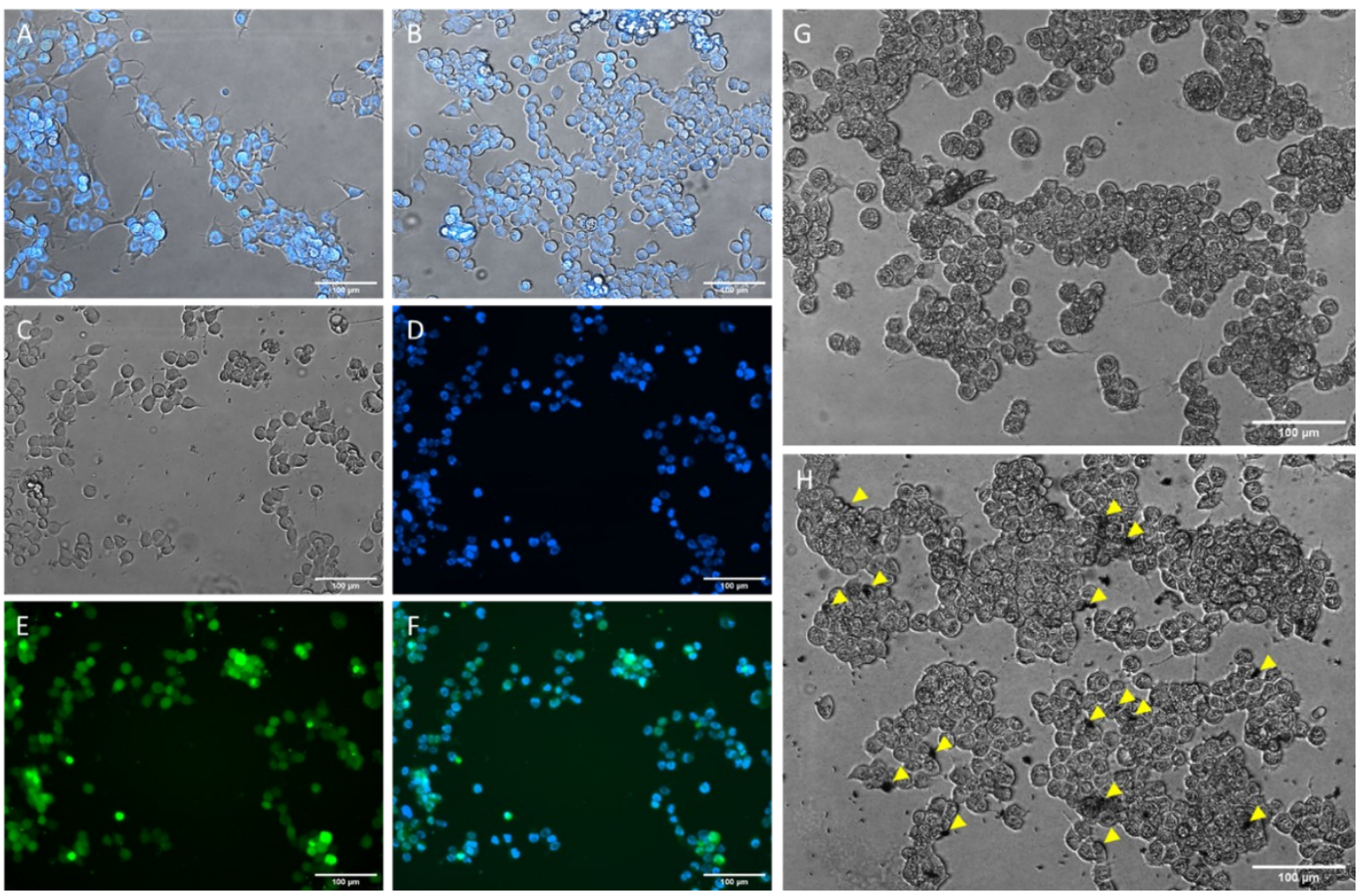

Figure 7 Wireless studies using NG108-15 cells. A-Cells before treatment with NucBlue stain (excitation/emission 360/460 nm). B-Cells after 4 hours submerged in PBS with NucBlue stain. C-F Cells after 4 hours of CNTP-6AF treatment, C- optical image, D-Nucblue fluorescent image, E- CNTP-6AF fluorescence (excitation/emission 490/520 nm), F- the composite image of $E \&$ E. G-Cells incubated with PBS for 4 hours then subjected to $2 \mathrm{~V}, \mathrm{DC}$, for 1 hour in $1 \mathrm{mM} \mathrm{AuCl}_{4}$ solution. $\mathrm{H}$-Cells incubated with CNTPs for 4 hours then subjected to $2 \mathrm{~V}, \mathrm{DC}$, for 1 hour in $1 \mathrm{mM} \mathrm{AuCl}_{4}$ solution. Yellow arrows indicate reduced gold deposits associated with the cellular membrane. All scale bars are $100 \mu \mathrm{M}$. 
Unlike the GUV samples which fused at higher voltages, the cells remain separated. Therefore, even if we hypothesized that the cell body was acting as a BPE rather than the individual CNTPs within its membrane, then according to Equation 1 we would need to be applying a driving potential of $800 \mathrm{~V}$. As less than $2 \mathrm{~V}$ was required, this observation supports the idea that the CNTPs are acting as individual nano-BPEs which do not follow the same rules as macro and micro BPEs. One reason why lower driving voltage is required at the nanoscale, especially, for electrodes with a radius below 50nm, is that the diffuse double layer extending from the CNTPs bipolar electrodes into the solution becomes significant ${ }^{46}$, and dramatically increases the capacitance associated with the charging current and at the CNTPs. Our observation of lower voltages required to initiate wireless electrochemistry at the nanoscale are also supported by our previous work on nano-BPEs ${ }^{54}$.

We also performed finite element modeling to further aid our understanding of bipolar electrochemistry at a CNTP embedded within a GUV (Figure 8). The model was based on the CNTP modified GUV with the CNTPs having a diameter of $0.8,2,5$ and $10 \mathrm{~nm}$ with a permittivity of 100 (semiconducting tubes) or2000 (metallic tubes) for CNTPs types, respectively, ${ }^{55}$ in a solution of $1 \mathrm{mM} \mathrm{AuCl}^{4-}$ and $\mathrm{H}^{+}$as the ions with a diffusion coefficient for $\mathrm{H}^{+}: 9.3 \times 10^{-9} \mathrm{~m}^{2} \mathrm{~s}^{-1}$ and for $\mathrm{AuCl} 4: 31.4 \times 10^{-12} \mathrm{~m}^{2} \mathrm{~s}^{-1}$. Calculated electric potential surface demonstrated that the applied potential propagated through the CNTP-lipid (Figure 8 A, B, and C). We calculated the electric potential distribution along the CNTP walls (Figure $8 \mathrm{D}$ ) and extracted the potential value at the position near the CNTP tips (Figure $8 \mathrm{E}$ ). The results demonstrated that there is a significant difference in the voltage density at the tip of the electrode at different diameter length scales. There is approximately a $30 \%$ increase in the induced voltage density at the pole on the CNTP when comparing the 1 $\mathrm{nm}$ diameter CNTPs to the $10 \mathrm{~nm}$ ones. This relationship in size in combination with the observation made by Compton et $\mathrm{al}^{46}$, explains why we are observing the electric field induced membrane electron transfers at potentials lower than predicted. This also indicates the theory on bipolar electrochemistry at the nanoscale needs to be modified to take account of the enhanced voltage gradient observed at the nanoscale which facilitated bipolar electrochemistry at the nanoscale. 

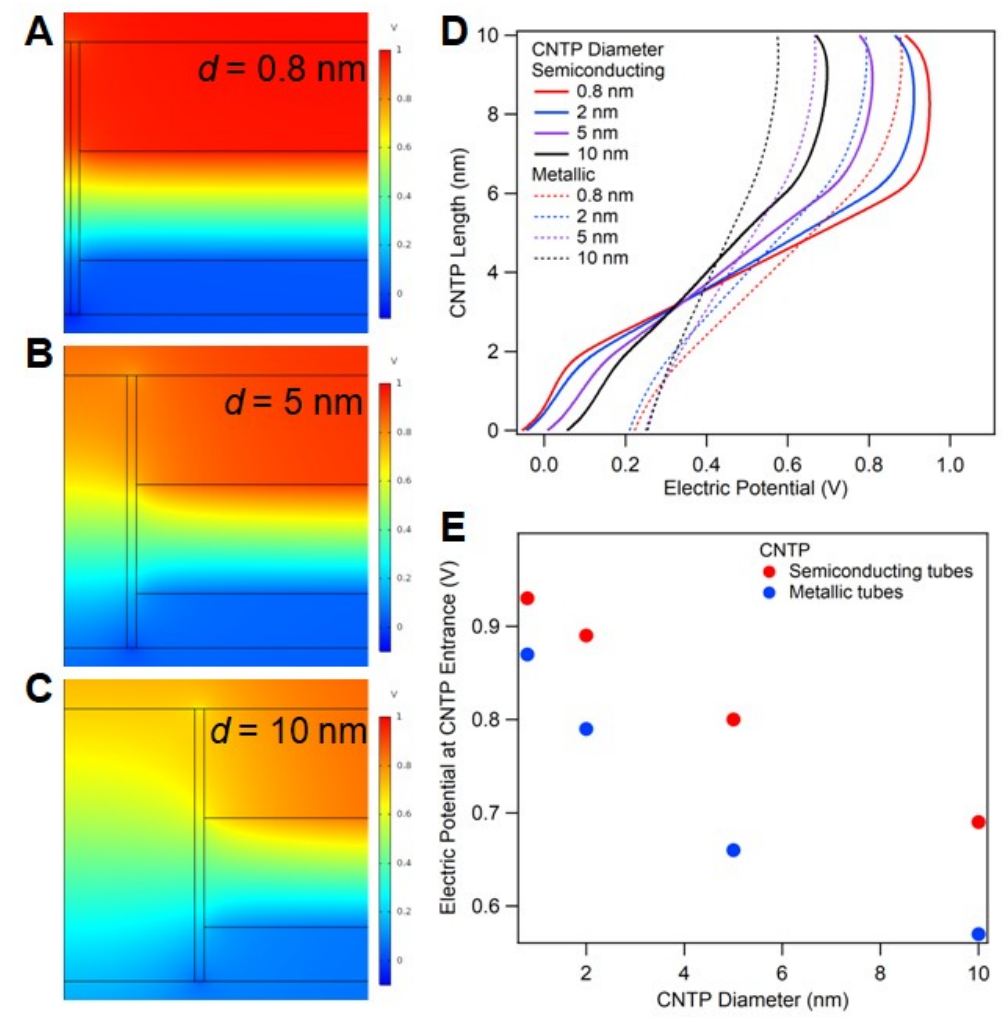

Figure 8. Modeled electric potential along the carbon nanotube porins (CNTPs) (A, B, C,D) and the electric potential values $(E)$ at the entrance when comparing CNTP diameters and electrical properties.

Even in light of these observations more work is needed to understand the exact relationship of capacitance at the nanoscale of BPEs in biology which we have begun to address ${ }^{56}$. However, we do show that nano-BPEs could be polarised at low, biocompatible voltages and we could modulate electron transfer across biological membranes and effect a change on membrane potential.

\section{Conclusion}

Here we have shown that CNTPs inserted and retained within lipids and plasma membranes of live cells where they can act as nano-BPEs. Despite their short size, the CNTPs can be polarised at low voltages (less than $2 \mathrm{~V}$ ) shown by the reduction of gold chloride to gold at the surface of the lipids and plasma membrane. This observation was confirmed by both conventional microscopy and SEM-EDX analysis. We observe similar gold reduction on the CNTP bipolar electrodes inserted into membranes of live cells, where the reduction of gold chloride was visible at $2 \mathrm{~V}$ applied potential. Such low, cell-friendly, voltages have only recently been theorized and shown and are unique to nano-BPEs with much larger voltages required for both micro and macro-sized BPEs. Our observations opens up the potential to use nano-BPEs in electronic therapeutics and in their development to help treat neurodegenerative diseases and cancer as artificial VDACs and TPMET mimics.

\section{Methods}

\section{Materials}

All materials unless otherwise stated were purchased from Sigma-Aldrich. 


\section{Carbon Nanotube Porin Synthesis}

The synthesis of CNTPs has been described in depth previously ${ }^{35}$. Briefly, $0.5 \mathrm{mg}$ single-walled carbon nanotubes (CNTs) were baked incrementally starting at $100^{\circ} \mathrm{C}$ and increasing temperature by $20^{\circ} \mathrm{C}$ every two minutes until a temperature of $475^{\circ} \mathrm{C}$ is reached. $28 \mathrm{mg}$ 1,2-dioleoyl-sn-glycero-3-phosphocholine (DOPC, Avanti Polar Lipids) was dried within a scintillation vial (VWR) using a RotaVapor Evaporator (Buchi). The baked CNTs were then solubilized with the dried DOPC with $14 \mathrm{mls}$ MilliQ water. The solution was placed in a sonicator water bath (Branson 1800) for approximately 30 minutes to fully solubilize the CNTs. The solution was then transferred to the ultrasonicator (QSonica) within a sound enclosure (QSonica) with a microtip diameter of 0.25 inches. The sample was held by a custom-made sample holder that allowed water circulation that was kept at a constant temperature of $30^{\circ} \mathrm{C}$ by a recirculating chiller (Polyscience). The sample was sonicated for a total of 16 hours by pulses (3s on, $1 \mathrm{~s}$ off) at an amplitude of $35 \%$. After sonication, the sample was centrifuged (Beckman Coulter with Allegra X-Rotor) for 1 hour at 10300g. The supernatant containing the CNTPs was then carefully aspirated for further use. Calculation of yield of CNTPs is measured as the number of CNTPs per vesicle via a proton assay and is described in depth here ${ }^{35}$.

\section{CNTP Modification and Purification}

A concentration of approximately $20 \mathrm{CNTPs} / \mathrm{vesicle} / \mathrm{ml}$ was dried in glass vials using a CentriVap micro-IR concentrator (Labconco). The dried CNTPs were then resolubilized in $1 \mathrm{ml}$ MilliQ water containing 80mM 1ethyl-3-(-3-dimethylaminopropyl) carbodiimide hydrochloride (EDC) and 20mM N-hydroxysuccinimide (NHS) and left at room temperature for 30 minutes. A saturated solution (approximately $25 \mathrm{mM}$ ) of 6aminofluorescein (6AF) was made in a solution mix of ethanol and water (50:50) and spun for 1 minute on a benchtop centrifuge to remove particulates. $100 \mu \mathrm{L}$ of the 6AF solution was added to the CNTPs giving a final concentration of approximately $2.5 \mathrm{mM}$. This solution was mixed gently by pipetting then allowed to react overnight at room temperature in the dark. The next day the bound CNTP-6AF was separated from unbound components by gel chromatography using a sepharose CL-6B column. Fractions were collected in a 96 well plate (Costar) and absorbance was measured in a Microplate reader (SpectraMax iD3 Multi-mode Microplate reader) at $1000 \mathrm{~nm}$ to detect CNTPs and $495 \mathrm{~nm}$ to detect 6AF. Selected fractions were then pooled and stored at $4^{\circ} \mathrm{C}$ until use.

\section{GUV Formation}

Giant unilamellar vesicles (GUVs) were formed using an adaptation of methods published previously by Orwar et al. ${ }^{57}$ and Manley and Gordon ${ }^{58}$. Lipids were dried within scintillation vials (VWR) containing $2 \mathrm{mg}$ DOPC and 0.02mg 1,2-dioleoyl-sn-glycero-3-phosphoethanolamine-N-(lissamine rhodamine B sulfonyl) (18:1 Liss Rhod PE, Avanti Polar Lipids) with a RotaVapor Evaporator (Buchi). The lipids were rehydrated with $1 \mathrm{ml}$ of CNTP-6AF (or $1 \mathrm{ml}$ water) and solubilized briefly in a water bath sonicator (Fisher Brand FB11021) before being left at room temperature for 30 minutes to form large unilamellar vesicles (LUVs). The LUV solution went through a process of freeze/thaw ten times using liquid nitrogen (BOC) to freeze and a water bath on a hot plate (VWR) set at $100^{\circ} \mathrm{C}$ to thaw the solution. This was done to break down double membranes produced when the LUVs were forming. 
Glass coverslips (13mm diameter, VWR) were cleaned by briefly sonicating (Fisher Brand FB11021) in acetone for 30 s followed by isopropanol for 10 s and rinsing with MilliQ water. Cleaned coverslips were dried with nitrogen gas. $5 \mu \mathrm{L}$ LUVs were pipetted onto the center of coverslips and dried under vacuum. Once dried coverslips were placed within a 12 well plate and rehydrated with $20 \mu \mathrm{L}$ for at least 10 minutes to allow the GUVs to form.

\section{Wireless Electrochemistry}

Wireless electrochemical experiments were conducted within a 24 well plate (Costar) with a custom-made (LLNL) well insert that could hold the driving electrodes at a set position and distance apart. Hypodermic needles were used as driving electrodes (25G, BD Microlance) and held in place by the insert. Dried LUVs were rehydrated to form GUVs for at least 10 minutes before electrical stimulation by the power source (Programmable AC/DC Power Source EC1000SA, NF Corporation). A direct current (DC) was used in all cases with the voltage applied varied between $1 \mathrm{~V}$ and $4 \mathrm{~V}$ and applied to the samples for 1 hour.

\section{Fluorescence Microscopy}

Images were captured with a Nikon fluorescent microscope (Model: TI-DH) with an optiMOS sCMOS camera and utilizing NIS-Elements (version 4.60) software.

\section{Confocal Microscopy}

Confocal microscopy was conducted using a Zeiss LSM 880 on a Zeiss Axio Observer platform system running Zen Black software. Z-Stacks were imaged with a Plan-Apochromat 20X/0.8 M27 objective with a $1 \mu$ M depth per slice and a total depth imaged of $20.35 \mu \mathrm{M}$. The pinhole was set to $0.73 \mathrm{AU}$ (for an excitation wavelength of $561 \mathrm{~nm}$ ) and $0.87 \mathrm{AU}$ (for an excitation wavelength of $488 \mathrm{~nm}$ ). The scanning was in unidirectional sequential line mode with four times averaging to reduce noise. Images were analyzed with a combination of ImageJ and Zen Blue.

\section{Scanning Electron Microscopy - Electron Dispersive X-Ray (SEM-EDX) Analysis}

Back-scattered electron (BSE) images and energy-dispersive X-ray spectroscopy (EDS) element maps were collected using a JEOL JSM-6490LV scanning electron microscope (SEM) with Oxford Instruments X-MAX INCA EDS system. The SEM was set to an accelerating voltage of $10 \mathrm{kV}$ and a specimen chamber pressure of $15 \mathrm{~Pa}$. Specimen regions with relatively high concentrations of carbon and gold were selected by mapping at low magnification, then rectangular areas $26 \mu \mathrm{m}$ by $20 \mu \mathrm{m}$ in size were mapped with a step size of $0.1 \mu \mathrm{m}$. The Xray energy window for the Au Ma line was adjusted to reduce overlap with the $\mathrm{P} K$ line.

\section{Cell Culture}

NG108-15 cells (ECACC) were cultured in dulbeccos modified media (Gibco) supplemented with 10\% FBS and $1 \%$ penicillin streptomycin. Cells were seeded in a 24 well plate (Costar, tissue culture treated) at 50,000 cells/ $\mathrm{ml}$ and grown for 2 days to reach confluency at $37^{\circ} \mathrm{C}$ and $5 \% \mathrm{CO}_{2}$. When confluent, media was carefully aspirated from wells by pipetting and replaced with either $0.5 \mathrm{ml}$ phosphate buffered saline (PBS) or modified 
CNTPs (CNTP-6AF) and returned to the incubator for 4 hours. After 4 hours the solution was replaced with $1 \mathrm{mM} \mathrm{AuCl}_{4}$ (in ultrapure MilliQ water) and wireless experiments conducted as described previously.

\section{Modeling}

To explore the applied electric potential on the CNTP, we modeled this system in the finite element analysis software COMSOL Multiphysics 5.5. Our simulation used two physical modules for describing the electric potential and the $\mathrm{AuCl}_{4}^{-}$distribution in this system: electrostatic and transport of diluted species. In this simplified model, we set up an axisymmetric cell that has two reservoirs with $50 \mathrm{~nm}$ in radius and height. These chambers were separated by lipid bilayers, which is $4 \mathrm{~nm}$ in thickness, and with a CNTP channel placed in the center. The external potential was applied at the upper boundary of the top reservoir and the bottom reservoir was grounded. $1 \mathrm{~V}$ was the voltage applied in this model system. The length of CNTP is $10 \mathrm{~nm}$, and the thickness of the CNTP wall is $0.34 \mathrm{~nm}$. The tips of CNTPs were assigned with a charge density equals to four electrons. $1 \mathrm{mM} \mathrm{AuCl}_{4}^{-}$was the starting concentration for both reservoirs. We varied the CNTP carbon-tocarbon diameters, $d$, with $0.8,2,5$, and $10 \mathrm{~nm}$ to probe the effect of tip size on electric potential distribution. We also altered the relative permittivity of CNTP using two values: 100 and 2000, which correspond to semiconducting tubes and metallic tubes, respectively.

\section{Acknowledgments}

This work was supported by the Engineering and Physical Sciences Research Council [Grant numbers EP/R004072/1, EP/L022494/1]. Parts of this work were performed at the Lawrence Livermore National Laboratory under the auspices of the U.S. Department of Energy under Contract DE-AC52-07NA27344, and were supported by the U.S. Department of Energy, Office of Basic Energy Sciences, Division of Materials Sciences and Engineering under Award SCW0972.

\section{References}

1. Principe, D. D.; Avigliano, L.; Savini, I.; Catani, M. V., Trans-Plasma Membrane Electron Transport in Mammals: Functional Significance in Health and Disease. Antiox. Red. Sig. 2011, 14 (11), 2289-2318.

2. Fischer, H., Mechanisms and function of DUOX in epithelia of the lung. Antiox. Redox. Signal. 2009, 11 (10).

3. $\quad$ Eccardt, A.; Bell, T.; Mattathil, L.; Prasad, R.; Kelly, S.; Fisher, J., Trans-Plasma Membrane Electron Transport and Ascorbate Efflux by Skeletal Muscle. Antiox 2017, 6 (4).

4. Ly, J.; Lawen, A., Transplasma membrane electron transport: enzymes involved and biological function. Redox report : communications in free radical research 2003, 8 (1).

5. Sarr, D.; Tóth, E.; Gingerich, A.; Rada, B., Antimicrobial actions of dual oxidases and lactoperoxidase. J. Microbiol. 2018, 56 (6), 373-386.

6. $\quad$ Principe, D. D.; Avigliano, L.; Savini, I.; Catani, M. V., Trans-Plasma Membrane Electron Transport in Mammals: Functional Significance in Health and Disease. Antiox. Redox. Signal. 2011, 14 (11), 2289-2318.

7. Block, M., NADPH oxidase as a therapeutic target in Alzheimer's disease. BMC neurosci 2008, 9 Suppl 2 (Suppl 2).

8. Jana, A.; Pahan, K., Fibrillar amyloid-beta peptides kill human primary neurons via NADPH oxidasemediated activation of neutral sphingomyelinase. Implications for Alzheimer's disease. The Journal of biological chemistry 2004, 279 (49).

9. $\quad$ Bruce-Keller, A.; Gupta, S.; Parrino, T.; Knight, A.; Ebenezer, P.; Weidner, A.; LeVine, H.; Keller, J.; Markesbery, W., NOX activity is increased in mild cognitive impairment. Antioxi \& Red Signal 2010, 12 (12). 
10. Robinson, A. J.; Jain, A.; Sherman, H. G.; Hague, R. J. M.; Rahman, R.; Sanjuan-Alberte, P.; Rawson, F. J., Toward Hijacking Bioelectricity in Cancer to Develop New Bioelectronic Medicine. Adv. Therap 2021, $n / a$ (n/a), 2000248.

11. Gibney, S.; Hicks, J. M.; Robinson, A.; Jain, A.; Sanjuan-Alberte, P.; Rawson, F. J., Toward nanobioelectronic medicine: Unlocking new applications using nanotechnology. WIREs Nanomed. Nanobiotech. 2021, $\mathrm{n} / \mathrm{a}$ (n/a), e1693.

12. Elinder, F.; Akanda, N.; Tofighi, R.; Shimizu, S.; Tsujimoto, Y.; Orrenius, S.; Ceccatelli, S., Opening of plasma membrane voltage-dependent anion channels (VDAC) precedes caspase activation in neuronal apoptosis induced by toxic stimuli. Cell. Death. Diff. 2005, 12 (8), 1134-1140.

13. Marin, R.; Ramírez, C.; González, M.; González-Muñoz, E.; Zorzano, A.; Camps, M.; Alonso, R.; Díaz, M., Voltage-dependent anion channel (VDAC) participates in amyloid beta-induced toxicity and interacts with plasma membrane estrogen receptor alpha in septal and hippocampal neurons. Mol. Mem. Biol. 2007, 24 (2), 148-60.

14. Yu, W.; Forte, M., Is there VDAC in cell compartments other than the mitochondria? J. Bioenerg. Biomem. 1996, 28 (2).

15. Mannella, C., Minireview: on the structure and gating mechanism of the mitochondrial channel, VDAC. J. Bioenerg. Biomem. 1997, 29 (6).

16. Okada, S.; O'Neal, W.; Huang, P.; Nicholas, R.; Ostrowski, L.; Craigen, W.; Lazarowski, E.; Boucher, R., Voltage-dependent anion channel-1 (VDAC-1) contributes to ATP release and cell volume regulation in murine cells. J. Gen. Phys. 2004, 124 (5).

17. Sherman, H.; Jovanovic, C.; Abuawad, A.; Kim, D.; Collins, H.; Dixon, J.; Cavanagh, R.; Markus, R.; Stolnik, S.; Rawson, F., Mechanistic insight into heterogeneity of trans-plasma membrane electron transport in cancer cell types. Biochim. Biophys. Acta. Bio. 2019, 1860 (8).

18. Stockwin, L.; Blonder, J.; Bumke, M.; Lucas, D.; Chan, K.; Conrads, T.; Issaq, H.; Veenstra, T.; Newton, D.; Rybak, S., Proteomic analysis of plasma membrane from hypoxia-adapted malignant melanoma. Journal of proteome research 2006, 5 (11), 2996-3007.

19. Ning, L.; Pan, B.; Zhao, Y.; Liao, Q.; Zhang, T.; Chen, G.; Wang, W.; Yang, Y., [Immuno-proteomic screening of human pancreatic cancer associated membrane antigens for early diagnosis]. Chi. J Surg 2007, 45 (1).

20. Akanda, N.; Tofighi, R.; Brask, J.; Tamm, C.; Elinder, F.; Ceccatelli, S., Voltage-dependent anion channels (VDAC) in the plasma membrane play a critical role in apoptosis in differentiated hippocampal neurons but not in neural stem cells. Cell cycle (Georgetown, Tex.) 2008, 7 (20), 3325-34.

21. Grupi, A.; Ashur, I.; Degani-Katzav, N.; Yudovich, S.; Shapira, Z.; Marzouq, A.; Morgenstein, L.; Mandel, Y.; Weiss, S., Interfacing the Cell with "Biomimetic Membrane Proteins". Small 2019, 15 (52), e1903006.

22. Fenno, L.; Yizhar, O.; Deisseroth, K., The development and application of optogenetics. Annu Rev Neurosci 2011, 34, 389-412.

23. Spangler, S. M.; Bruchas, M. R., Optogenetic approaches for dissecting neuromodulation and GPCR signaling in neural circuits. Curr. Opin. Pharmacol. 2017, 32, 56-70.

24. Simon, D. T.; Gabrielsson, E. O.; Tybrandt, K.; Berggren, M., Organic Bioelectronics: Bridging the Signaling Gap between Biology and Technology. Chem. Rev. 2016, 116 (21), 13009-13041.

25. Löffler, S.; Libberton, B.; Richter-Dahlfors, A., Organic Bioelectronic Tools for Biomedical Applications. Electronics 2015, 4 (4), 879-908.

26. Famm, K.; Litt, B.; Tracey, K. J.; Boyden, E. S.; Slaoui, M., Drug discovery: a jump-start for electroceuticals. Nature 2013, 496 (7444), 159-61.

27. Ma, Y.; Bao, J.; Zhang, Y.; Li, Z.; Zhou, X.; Wan, C.; Huang, L.; Zhao, Y.; Han, G.; Xue, T., Mammalian Near-Infrared Image Vision through Injectable and Self-Powered Retinal Nanoantennae. Cell 2019, 177 (2), 243-255.

28. Sushnitha, M.; Evangelopoulos, M.; Tasciotti, E.; Taraballi, F., Cell Membrane-Based Biomimetic Nanoparticles and the Immune System: Immunomodulatory Interactions to Therapeutic Applications. Frontiers in bioengineering and biotechnology 2020, 8.

29. Nednoor, P.; Gavalas, V. G.; Chopra, N.; Hinds, B. J.; Bachas, L. G., Carbon nanotube based biomimetic membranes: mimicking protein channels regulated by phosphorylation. J. Mat Chem. 2007, (18).

30. García-Fandiño, R.; Sansom, M. S. P., Designing biomimetic pores based on carbon nanotubes. Period. Nat. Acad. Sci. 2012, 109 (18), 6939-6944.

31. Gupta, A. K.; Naregalkar, R. R.; Vaidya, V. D.; Gupta, M., Recent advances on surface engineering of magnetic iron oxide nanoparticles and their biomedical applications. Nanomedicine 2007, 2 (1), $23-39$. 
32. Weingart, J.; Vabbilisetty, P.; Sun, X. L., Membrane mimetic surface functionalization of nanoparticles: methods and applications. Adv Colloid Interface Sci 2013, 197-198, 68-84.

33. Park, K.; Kuo, Y.; Shvadchak, V.; Ingargiola, A.; Dai, X.; Hsiung, L.; Kim, W.; Zhou, H.; Zou, P.; Levine, A.; Li, J.; Weiss, S., Membrane insertion of-and membrane potential sensing by-semiconductor voltage nanosensors: Feasibility demonstration. Sci. Adv. 2018, 4 (1).

34. Rothemund, P., Folding DNA to create nanoscale shapes and patterns. Nature 2006, 440 (7082), 297-

302.

35. Tunuguntla, R. H.; Escalada, A.; Frolov, V. A.; Noy, A., Synthesis, lipid membrane incorporation, and ion permeability testing of carbon nanotube porins. Nat. Prot. 2016, 11 (10), 2029.

36. Hodge, T.; Colombini, M., Regulation of metabolite flux through voltage-gating of VDAC channels. J. Mem. Biol. 1997, 157 (3), 271-9.

37. Li, Y.; Li, Z.; Aydin, F.; Quan, J.; Chen, X.; Yao, Y.-C.; Zhan, C.; Chen, Y.; Pham, T. A.; Noy, A., Waterion permselectivity of narrow-diameter carbon nanotubes. Sci. Adv. 2020, 6 (38).

38. Sanborn, J.; Chen, X.; Yao, Y.; Hammons, J.; Tunuguntla, R.; Zhang, Y.; Newcomb, C.; Soltis, J.; De, Y., JJ; Van, B., A; Parikh, A.; Noy, A., Carbon Nanotube Porins in Amphiphilic Block Copolymers as Fully Synthetic Mimics of Biological Membranes. Adv. Mat. 2018, 30 (51).

39. Wang, J. Z.; Ding, Z. Q.; Zhang, F.; Ye, W. B., Recent development in cell encapsulations and their therapeutic applications. Mater Sci Eng C Mater Biol Appl 2017, 77, 1247-1260.

40. Koefoed, L.; Pedersen, S.; Daasbjerg, K., Bipolar electrochemistry- A wireless approach for electrode reactions. Cur. Opin. Electrochem. 2017, 2 (1), 13-17.

41. Warakulwit, C.; Nguyen, T.; Majimel, J.; Delville, M. H.; Lapeyre, V.; Garrigue, P.; Ravaine, V.; Limtrakul, J.; Kuhn, A., Dissymmetric carbon nanotubes by bipolar electrochemistry. Nano. Lett. 2008, 8 (2), 500-4.

42. Karimian, N.; Hasemi, P.; Afkhami, A.; Bagheri, H., The principles of bipolar electrochemistry and its electroanalysis applications. Cur. Opin. Electrochem. 2019, 17, 30-37.

43. Guo, Q.; Lei, C.; Chen, W.; Zhang, J.; Huang, B., Electric field driven nanoparticles produce dual functional bipolar electrodes and nanoelectrolytic cells for remediation. Cell Rep. Phys. Sci. 2021.

44. Hao, R.; Fan, Y.; Han, C.; Zhang, B., Bipolar Electrochemistry on a Nanopore-Supported Platinum Nanoparticle Electrode. Anal. Chem. 2017, 89 (23), 12652-12658.

45. Ino, K.; Yaegaki, R.; Hiramoto, K.; Nashimoto, Y.; Shiku, H., Closed Bipolar Electrode Array for OnChip Analysis of Cellular Respiration by Cell Aggregates. ACS Sens 2020, 5 (3), 740-745.

46. Dickinson, E.; Compton, R., Diffuse double layer at nanoelectrodes. J Phys Chem C 2009, 113 (41), 17585-17589.

47. Fenz, S. F.; Sengupta, K., Giant vesicles as cell models. Integ. Biol. 2012, 4 (9), 982-995.

48. Baumgart, T.; Hammond, A. T.; Sengupta, P.; Hess, S. T.; Holowka, D. A.; Baird, B. A.; Webb, W. W., Large-scale fluid/fluid phase separation of proteins and lipids in giant plasma membrane vesicles. PNAS 2007, 104 (9), 3165-3170.

49. Duval, J.; Kleijn, J.; Leeuwen, H., Bipolar electrode behaviour of the aluminium surface in a lateral electric field. J .Electroanal. Chem 2001, 505 (1-2), 1-11.

50. and, J. F. L. D.; Leeuwen, H. P. v.; Cecilia, J.; Galceran, J., Rigorous Analysis of Reversible Faradaic Depolarization Processes in the Electrokinetics of the Metal/Electrolyte Solution Interface. J. Phys. Chem. B 2003, 107 (28), 6782-6800.

51. Duval, J. F. L.; M. Minor; J. Cecilia, a.; Leeuwent, H. P. v., Coupling of Lateral Electric Field and Transversal Faradaic Processes at the Conductor/Electrolyte Solution Interface. J. Phys, Chem. 2003, 107 (17), 4143-4155.

52. Standard Reduction Potential. https://chem.libretexts.org/@go/page/282 (accessed March 25).

53. J, G.; K, K.; J, Z.; A, E.; R, T.; LR, C.; FI, A.; AV, S.; KR, C.; D, M.; YM, W.; CP, G.; CM, A.-F.; VA, F.; A, N., Stochastic transport through carbon nanotubes in lipid bilayers and live cell membranes. Nature 2014, 514 (7524).

54. Sanjuan-Alberte, P.; Jain, A.; Shaw, A.; Abayzeed, S.; Dominguez, R.; Alea-Reyes, M.; Clark, M.; Alexander, M.; Hague, R.; Perez-Garcia, L.; Rawson, F., Wireless nanobioelectronics for electrical intracellular sensing. ACS App. Nanomat. 2019, 2 (10), 6397-6408.

55. Lu, W.; Wang, D.; Chen, L., Near-static dielectric polarization of individual carbon nanotubes. Nano. Lett. 2007, 7 (9), 2729-2733.

56. Robinson, A.; Jain, A.; Rahman, R.; Abayzeed, S.; Hague, R.; Rawson, F., Impedimetric characterisation of bioelectronic nano-antennae. ChemRxiv 2020, Preprint. 
57. Jesorka, A.; Stepanyants, N.; Zhang, H.; Ortmen, B.; Hakonen, B.; Orwar, O., Generation of phospholipid vesicle-nanotube networks and transport of molecules therein. Nat Protoc 2011, 6 (6), 791-805.

58. Manley, S.; Gordon, V. D., Making giant unilamellar vesicles via hydration of a lipid film. Curr. Protoc. Cell. Biol. 2008, Chapter 24, Unit 24.3.

59. Mueller, P.; Chien, T. F.; Rudy, B., Formation and properties of cell-size lipid bilayer vesicles. Biophys J 1983, 44 (3), 375-81. 


\section{Supporting information}

\section{Electric Field Induced Biomimetic Transmembrane Electron Transport using Carbon Nanotube Porins as Bipolar Electrodes:}

Jacqueline M. Hicks ${ }^{1}$, Yun-Chiao Yao ${ }^{2}$, Sydney Barber ${ }^{3,4}$, Nigel Neate ${ }^{5}$, Julie A. Watt ${ }^{1}$, Aleksandr Noy ${ }^{3}$, Frankie J. Rawson ${ }^{* 1}$

1-Biodiscovery Institute, School of Pharmacy, Division of Regenerative Medicine and Cellular Therapies, University of Nottingham, UK, NG7 2RD

2- School of Natural Sciences, University of California Merced, Merced, California 95344, United States Yun-Chiao Yao 3- Materials Science Division, Lawrence Livermore National Laboratory, USA, 94550 4-United States Naval Academy, USA, 21402

5-Nanoscale and Microscale Research Centre, University of Nottingham, UK, NG7 2RD

*Email: frankie.rawson@nottingham.ac.uk

\section{CNTP-6AF Binding}

The Raman spectra were measured using a Nicolet Almega XR micro-Raman spectrometer at laser wavelengths of $633 \mathrm{~nm}$. The CNTP samples (ca. $10 \mu \mathrm{L}$ ) were dried on glass slides in a vacuum desiccator for $1 \mathrm{hr}$ to form a film spot for Raman measurements. The laser power used was set less than $10 \%\left(100 \mathrm{~W} / \mathrm{cm}^{2}\right)$ to avoid heating the CNTP sample.

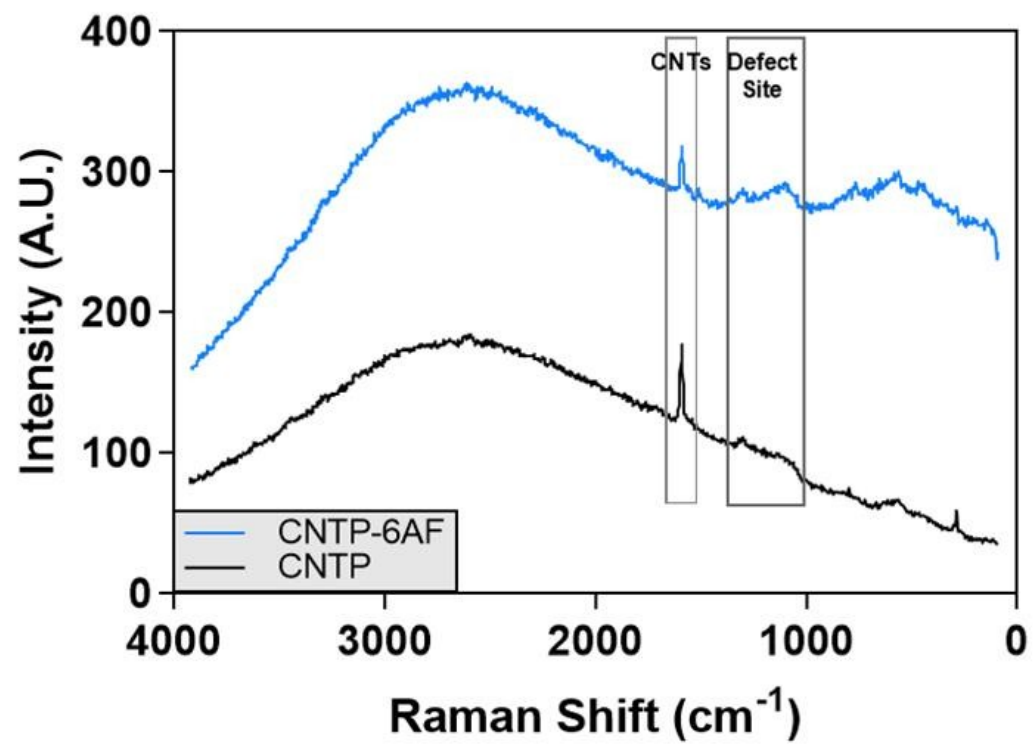

SI 1 Raman spectra of CNTP and CNTPs modified with 6-amino fluorescein (6AF). Regions highlighted indicated peaks assigned to either the CNTs or the defect site of the CNTs where the modification occurs. 
Fourier transform infrared spectroscopy (FTIR) was conducted on using an Agilent Cary 630 with ATR attachment. Samples (ca. $10 \mu \mathrm{l}$ ) were pipetted onto glass slides that had been cleaned with acetone then dried in a vacuum desiccator for 1 hour. Multiple layers (up to 5) of sample were dried in order to increase the concentration on the surface with the contact of the ATR attachment made at the edge of the dried area where concentration was greatest.

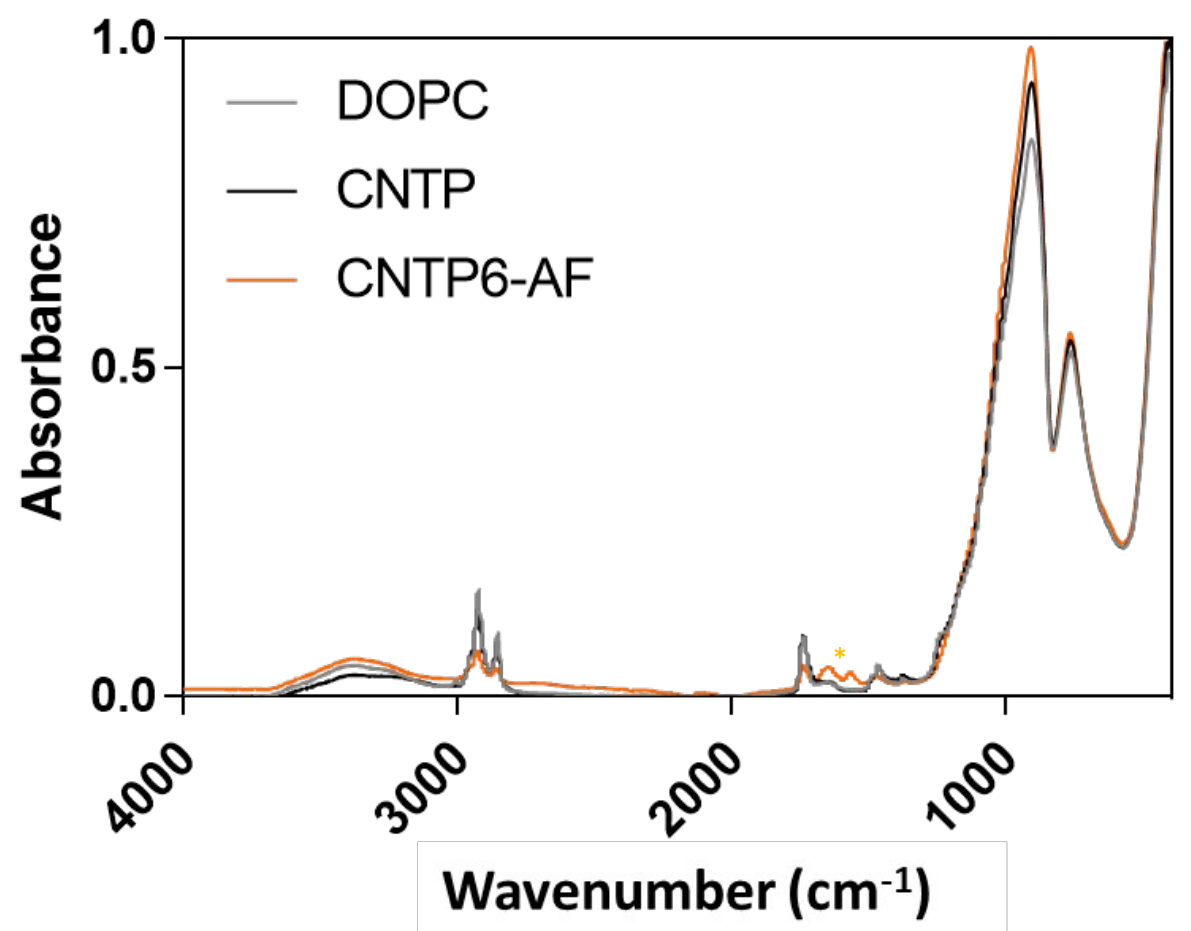

SI 2 FTIR Spectra of DOPC lipid, CNTP and CNTP modified with 6 amino fluorescein (6AF). Additional peaks found in the CNTP6-AF sample are indicated by the orange * and are in the NH range.

\section{GUV Formation}

Giant unilamellar vesicles (GUVs) were formed from large unilamellar vesicles (LUVs) as described previously in the literature ${ }^{57-59}$. This involved the formation of LUVs in solution which were then dried onto a surface under vacuum before being rehydrated with water. This rehydration step allows the LUVs to fuse into GUVs, attached to the surface by ionic interactions, within minutes. GUVs made with varying concentrations of lipid were then analysed by brightfield imaging GUVs form within minutes of rehydration and the composition of the lipids can be altered to the requirements. The lipid content here was comprised of 98\% DOPC (1,2-dioleoyl-sn-glycero-3phosphocholine) and 2\% 18:1 Liss Rhod PE (1,2-dioleoyl-sn-glycero-3-phosphoethanolamine-N-(lissamine rhodamine $B$ sulfonyl)) to allow the detection of the lipids by fluorescence. 


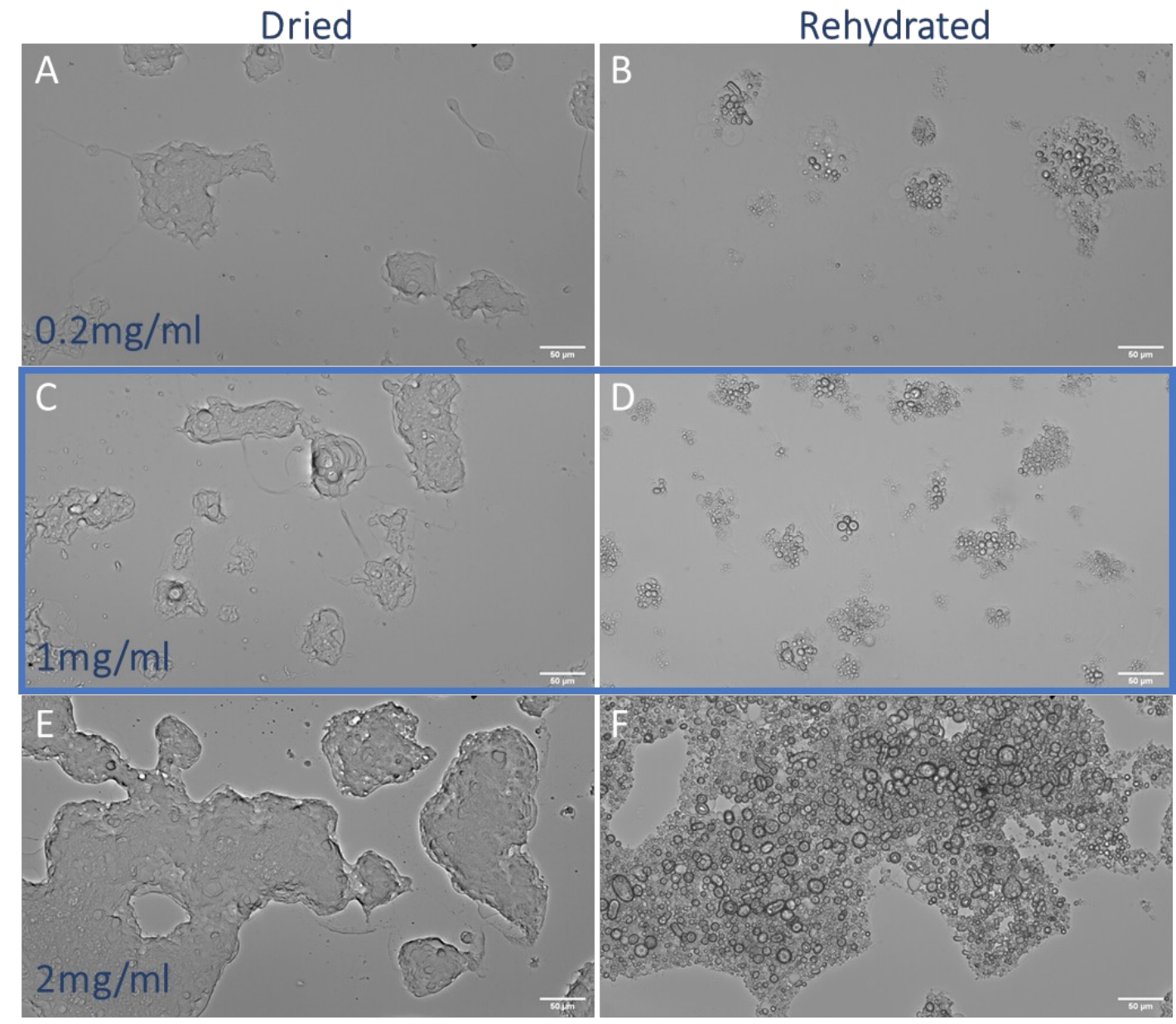

SI 3 Brightfield images of DOPC before and after rehydration at 10X magnification. Left hand column show DOPC dried onto the base of a 24 well plate $(5 \mu \mathrm{L})$ while right hand column shows DOPC after rehydration with $20 \mu \mathrm{L}$ water. A and B have a concentration of $0.2 \mathrm{mg} / \mathrm{ml}$ of DOPC, $C$ and $D 1 \mathrm{mg} / \mathrm{ml}$ and $E$ and $\mathrm{F}$ have $2 \mathrm{mg} / \mathrm{ml}$. Scale bars show $50 \mu \mathrm{m}$. Highlighted panels indicate the $1 \mathrm{mg} / \mathrm{ml}$ concentration that was chosen for future experiments.

\section{Imaging of CNTPs by cryo-TEM}

Samples were prepared using a Gatan CP3 Cryoplunge providing a controlled environment (70 - $80 \%$ humidity, $18^{\circ} \mathrm{C}$ ), by depositing $3 \mu \mathrm{L}$ of sample onto a TEM grid (300 mesh Cu, holey carbon or holey carbon / graphene oxide support film, EM Resolutions Ltd). Samples were blotted (1.5 s) before plunging into liquid ethane to vitrify. Samples were maintained under liquid nitrogen $\left(-196^{\circ} \mathrm{C}\right)$ until transfer to a TEM cryo sample holder (Gatan 926) and held at or below $-160{ }^{\circ} \mathrm{C}$ during analysis (Gatan Smartset 900). TEM images were recorded an a JEOL 2100 Plus, operating at $200 \mathrm{kV}$ using a Gatan Ultrascan 100XP camera. 

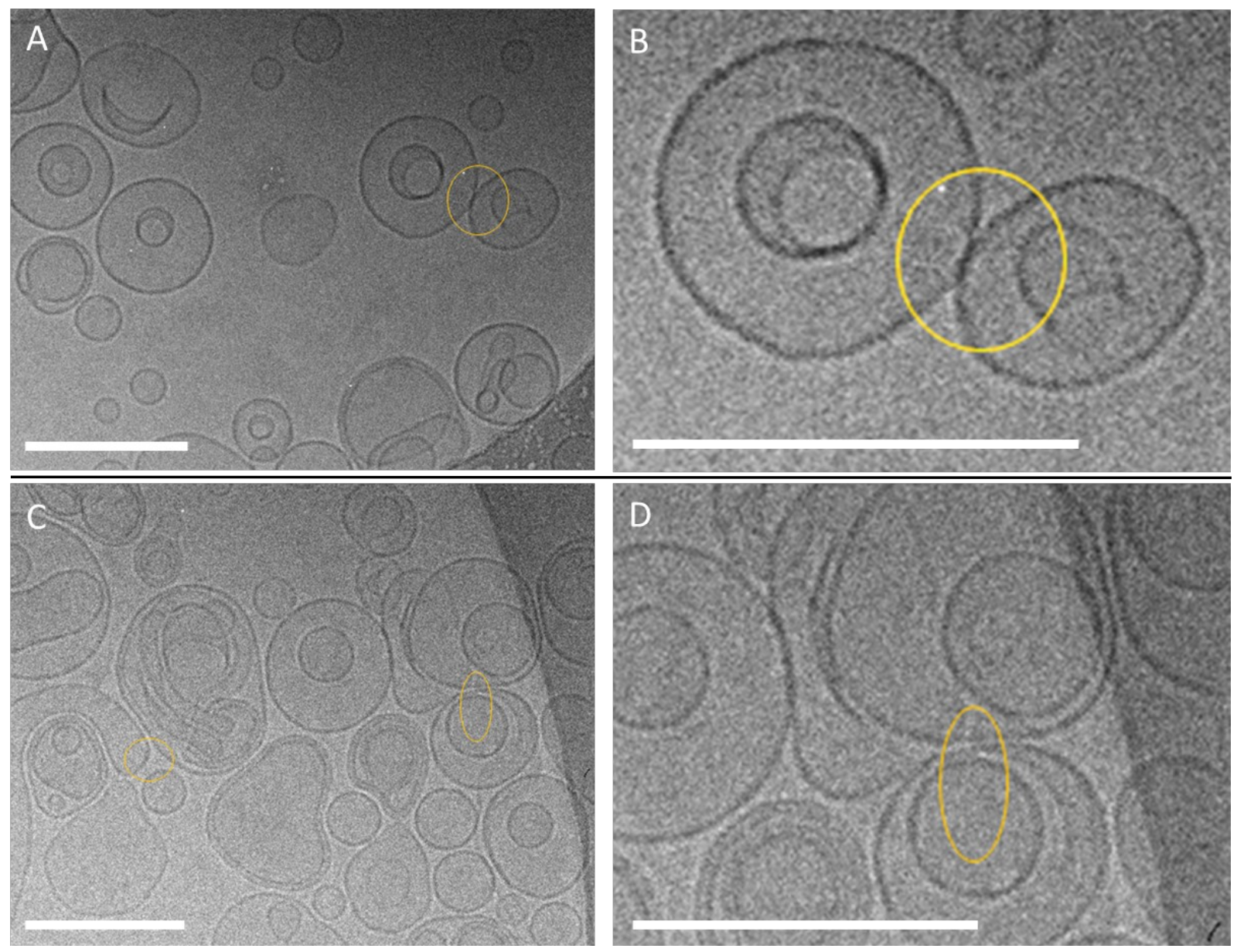

SI 4 Cryo-TEM images of GUVs with CNTPs within the membrane. Panel B is a zoomed in section of panel A while D is a zoomed in section of panel C. All scale bars are $200 \mu \mathrm{M}$. CNTs visible within the membrane are circled.

The CNT structures highlighted in figure SI 4 are measured to be approximately $23 \mathrm{~nm} \times 1.6 \mathrm{~nm}$ (length $\mathrm{x}$ width) and $28 \mathrm{~nm} \times 2.5 \mathrm{~nm}$; the single CNT structure in SI 4D is measured to be between 28 and $40 \mathrm{~nm}$ long and $3.4 \mathrm{~nm}$ wide. Given the low resolution of the images at that magnification and the distribution of CNT lengths that will be present within a sample these values align with the dimensions of CNTPs which are reported as minimum $0.7 \mathrm{~nm}$ wide and approximately $10 \mathrm{~nm}$ in length ${ }^{35}$. 


\section{Threshold Potential Application to Neuronal Cells}
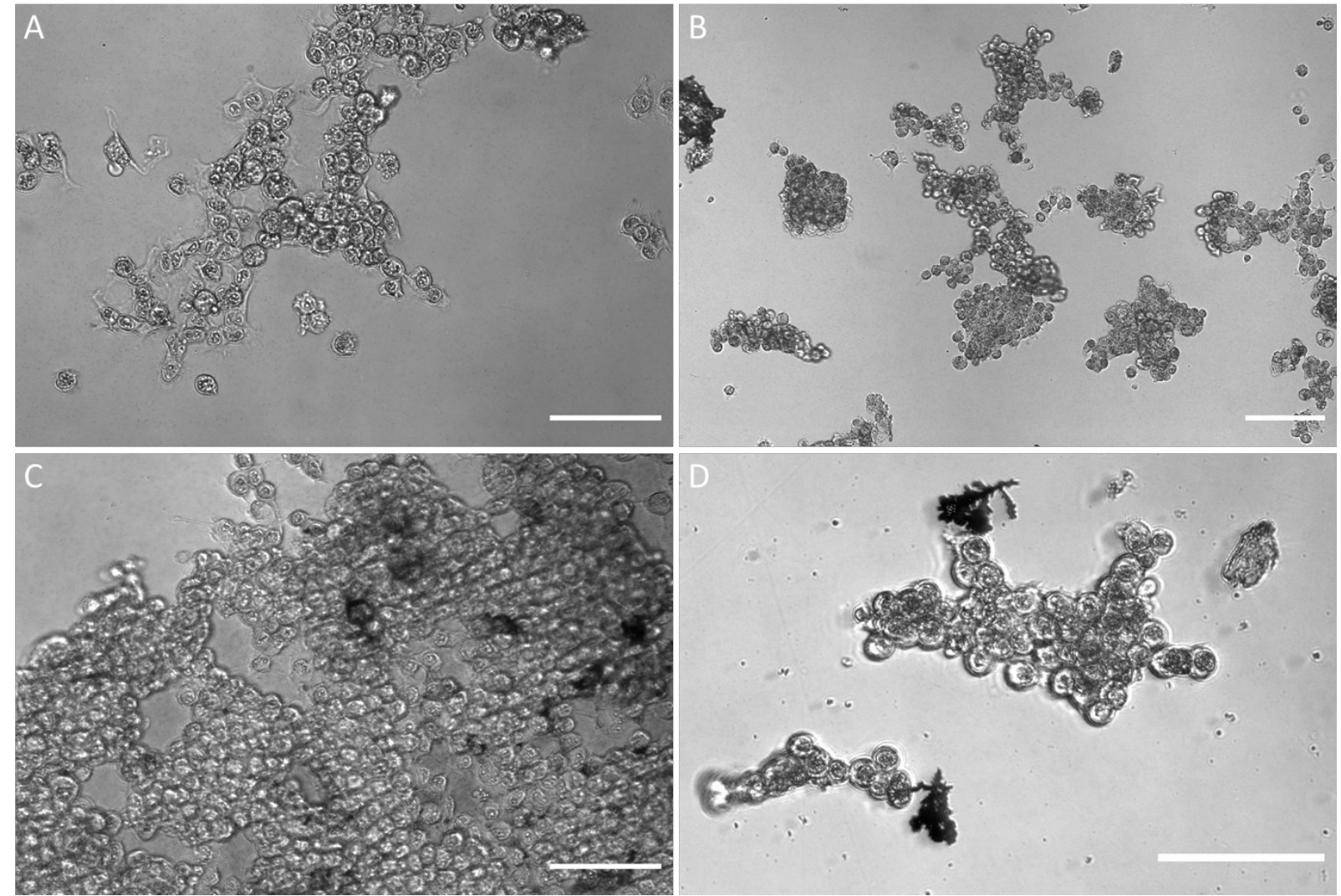

SI 5 NG108-15 cells stimulated at $1.5 \mathrm{~V}$ DC for one hour while submerged in $1 \mathrm{mM} \mathrm{AuCl}_{4}$ (in water) Panels $A \& B$ are of cells that were incubated with PBS for 4 hours. Panels $C$ and D are of cells incubated with CNTP-6AF for 4 hours. Scale bars are $100 \mu \mathrm{M}$.

\section{High Voltage Stimulation with Zinc Chloride}

Giant unilamellar vesicles (GUVs) were prepared as described in the main text. As an alternative to gold chloride, zinc chloride was used for metal deposition triggered by the current density at the CNT terminus. Concentration was normalised to take into account the difference in ionic concentration and a higher voltage of $7 \mathrm{~V}$ was required to trigger to zinc deposition; this is most likely due zinc being approximately three times more resistive than gold. The inset images show the stark differences in zinc deposition within the solution that is visible by eye while the brightfield images show deposits associated with the lipid membranes when CNTPs are present within the membrane (SI 6 B and C) whereas no membrane-associated deposits are seen in the control (SI $6 \mathrm{~A})$. 

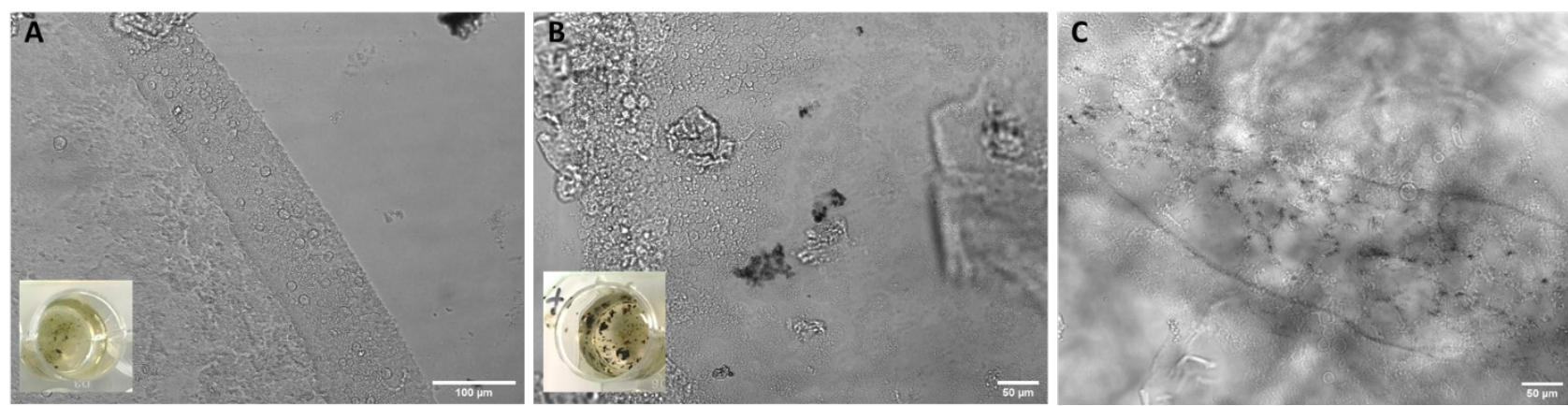

SI 6 Brightfield images of GUVs either without CNTPs (A) or containing CNTPs (B) and (C) that were submerged in $2.5 \mathrm{mM}$ $\mathrm{ZnCl}_{2}$ and stimulated with $7 \mathrm{~V}$ for 1 hour. (A) and (B) inset images show photographs of the wells immediately after stimulation. Scale bars are $50 \mu \mathrm{M}$ in length.

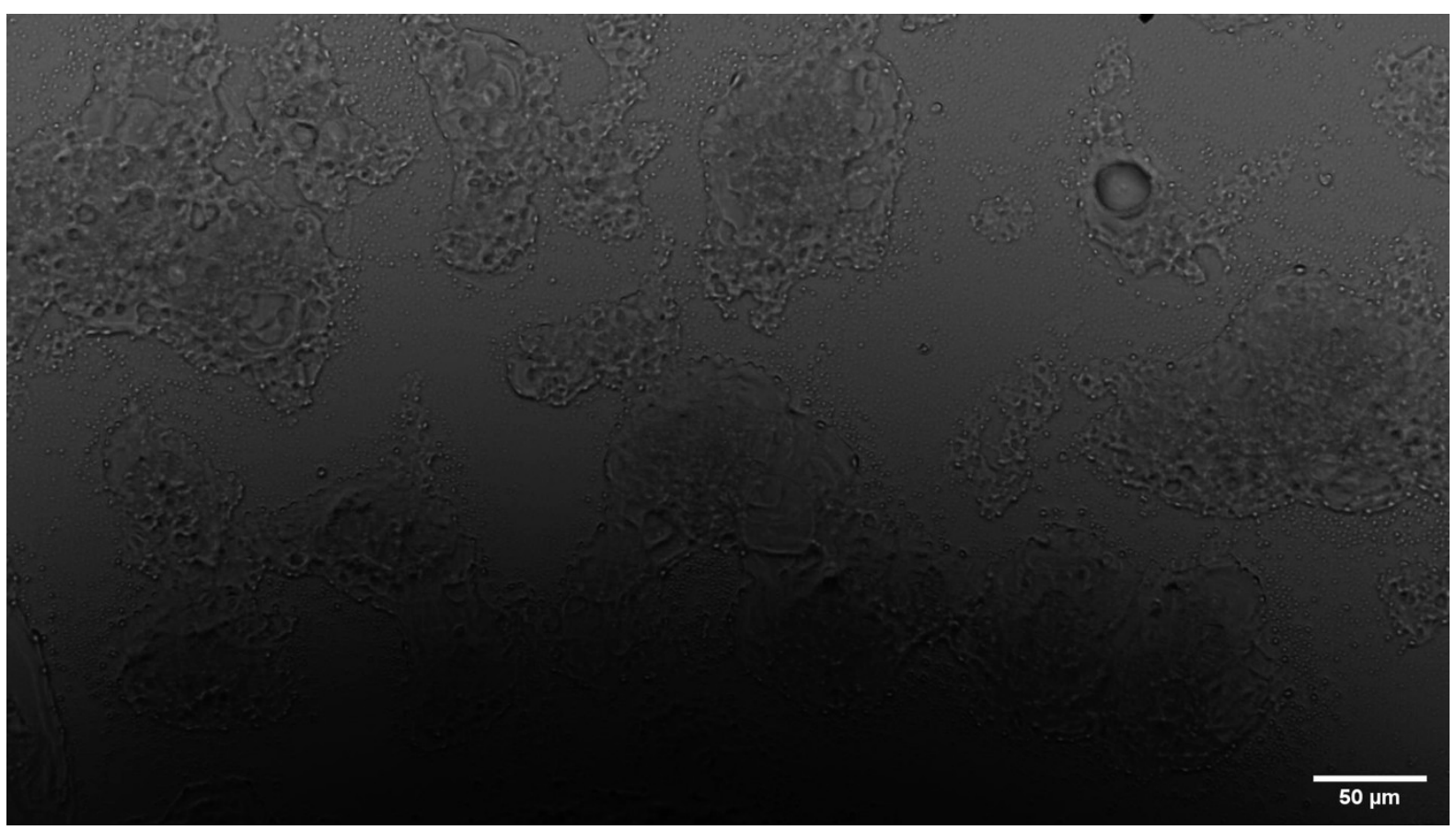

Si 7: 'Time lapse of GUVs modified with carbon nanotube porins stimulated at $1.5 \mathrm{~V}$ DC submerged in $1 \mathrm{mM}$ $\mathrm{AuCl}_{4}$ (in water)

1. Manley, S.; Gordon, V. D., Making giant unilamellar vesicles via hydration of a lipid film. Curr Protoc Cell Biol 2008, Chapter 24, Unit 24.3.

2. Mueller, P.; Chien, T. F.; Rudy, B., Formation and properties of cell-size lipid bilayer vesicles. Biophys J 1983, 44 (3), 375-81.

3. Jesorka, A.; Stepanyants, N.; Zhang, H.; Ortmen, B.; Hakonen, B.; Orwar, O., Generation of phospholipid vesicle-nanotube networks and transport of molecules therein. Nat Protoc 2011, 6 (6), $791-805$. 
4. Tunuguntla, R. H.; Escalada, A.; Frolov, V. A.; Noy, A., Synthesis, lipid membrane incorporation, and ion permeability testing of carbon nanotube porins. Nat. Prot. 2016, 11 (10), 2029. 